\title{
DIE WAHL DER RICHTER AM DEUTSCHEN BUNDESVERFASSUNGSGERICHT ZWISCHEN RECHTLICHER THEORIE UND POLITISCHER PRAXIS
}

Uwe Kischel*

\section{WÄHLBARKEIT}

Zum Richter am Bundesverfassungsgericht ist nur wählbar, wer das 40. Lebensjahr vollendet hat, zum Bundestag wählbar ist, schriftlich seine Bereitschaft zur Amtsübernahme erklärt und die Befähigung zum Richteramt besitzt, § 3 Abs. 1, 2 BVerfGG. Die Bereitschaftserklärung ist bedingungs- und befristungsfeindlich, doch kann sie - im erst-rechtSchluß aus $\S 12$ BVerfGG - jederzeit zurückgenommen werden. Als Zeitpunkt, zu dem diese Voraussetzungen vorliegen müssen, werden die Aufnahme in die beim Bundesministerium der Justiz zu führenden Vorschlagslisten, die Wahl oder die Ernennung in Betracht gezogen ${ }^{1}$. Die Vorschlagslisten besitzen jedoch für die Wahl keinen bindenden Charakter $^{2}$, so daß auf sie jedenfalls nicht abzustellen ist. Für den Zeitpunkt der Ernennung spricht, daß der Gewählte mit inr zum Richter wird und § 3 BVerfGG vom Wortlaut her nicht auf die Wahl selbst, sondern auf die Richtereigenschaft abstellt ("Die Richter müssen ..."). Doch wäre es, auch im Sinne der Rechtssicherheit, kaum sinnvoll, wenn der Bundestag eine Person wählte, die im Zeitpunkt der Wahl nicht die gesetzlichen Voraussetzungen erfüllte, deren mögliche Ernennung damit unsicher wäre. Die Voraussetzungen sollten daher bereits - aber auch erst - zum Zeitpunkt der Wahl vorliegen.

Daß Verfassungsrichter die Befähigung zum Richteramt i.S.v. §§ 5 Abs. 1, 7 DRiG haben müssen, markiert die entstehungsgeschichtlich ${ }^{3}$, rechtsvergleichend und insbesondere im Gegensatz zu einigen Landesverfassungsgerichten markante Entscheidung für ein reines Juristengericht. Sie gibt einen ersten Hinweis darauf, daß die Tätigkeit des Bundesverfassungsgerichts als eine rechtliche, die Anwendung der Verfassung als juristischer Erkenntnis- und Wertungsakt angesehen wird, der von Laien, etwa verdienten und erfahrenen Politikern, nicht angemessen zu bewältigen ist. Diese anfängliche Weichenstellung wurde in der weiteren Entwicklung bekräftigt. Nicht nur ist seit langem keine Kritik mehr an der notwendigen Befähigung zum Richteramt zu hören. Auch ist das 1951 noch bestehende, zusätzliche Qualifikationsmerkmal, demzufolge die Richter "im öffentlichen Leben erfahren" sein mußten, später gestrichen worden ${ }^{4}$. Die Forderung Wiltraut Rupp-von Brünnecks, neben Juristen müßten auch Personen aus verantwortlichen Positionen mit vertieften Kenntnissen des politischen Raumes am Gericht vertreten sein ${ }^{5}$, fand kein Gehör. Dem Gericht gelang es offenbar, den Eindruck zu vermeiden, es berücksichtige den "politischen Raum" ${ }^{6}$ nicht hinreichend.

Spezielle Wählbarkeitsvoraussetzungen bestehen für eine Untergruppe der Verfassungsrichter, nämlich für die drei Richter an jedem Senat, die nach § 2 Abs. 3 BVerfGG aus der Zahl der Richter an den obersten Gerichtshöfen des Bundes i.S.v. Art. 95 Abs. 1 GG gewählt werden müssen. Die einfachrechtliche Vorgabe beruht auf Art. 94 Abs. 1 S. 1 GG, der selbst aber nur mindestens zwei Bundesrichter und mindestens zwei andere Mitglieder verlangt. Die Bundesrichter sollen den ihnen eigenen besonderen Erfahrungsschatz und die damit verbundenen Kenntnisse und Fähigkeiten in die Senate einbringen. Diese Zwecksetzung reflektiert auch $\S 2$ Abs. 3 S. 2 BVerfGG, dem zufolge nur Bundesrichter mit mindestens dreijähriger Amtstätigkeit gewählt werden sollen ${ }^{7}$. Damit wird einer pro-forma Ernennung zum Bundesrichter zwecks Wahl zum Verfassungsrichter vorgebeugt. Allerdings handelt es sich um eine bloße Sollvorschrift ${ }^{8}$.

$\S 2$ Abs. 3 BVerfGG formuliert keine Obergrenze; in der Praxis war die Zahl der Bundesrichter durchaus auch einmal höher ${ }^{8}$. Dadurch kann es allerdings zu einem Problem kommen: Muß der direkte Nachfolger eines in seiner Eigenschaft als Bundesrichter gewählten Verfassungsrichters selbst dann Bundesrichter sein, wenn auch anderenfalls im jeweiligen Senat mindestens drei Verfassungsrichter ehemalige Bundesrichter sind? Die beiden möglichen Antworten auf diese praktisch bedeutsame und früher zum Teil mit Verve diskutierte Frage führen jeweils zu einem in sich folgerichtigen, geschlossenen Regelkomplex: Einerseits kann von zwei streng getrennten Gruppen der als Bundesrichter und der nicht als 


\section{DIE WAHL DER RICHTER AM DEUTSCHEN BUNDESVERFASSUNGSGERICHT ZWISCHEN RECHTLICHER THEORIE UND POLITISCHER PRAXIS}

Bundesrichter gewählten Verfassungsrichter ausgegangen werden. Dann muß nicht nur - in Beantwortung der Ausgangsfrage - zum Nachfolger eines Bundesrichters stets wieder ein Bundesrichter gewählt werden. Auch müßte zur Proporzwahrung im Rahmen des $\S 5$ Abs. 1 BVerfGG grundsätzlich das Wahlorgan von Nachfolger und Vorgänger identisch sein. Selbst die Vorschrift des $\S 101$ Abs. 1 BVerfGG i.V.m. $\S 70$ DRiG, wonach bei früheren Bundesrichtern mit der Ernennung das frühere Amt ausnahmsweise nicht endet sondern ruht, gält nur für gruppenangehörige Bundesrichter ${ }^{9}$. Andererseits kann anstelle dieser formalen Gruppenbetrachtung auch allein danach gefragt werden, ob ein Verfassungsrichter rein tatsächlich zuvor Bundesrichter war ${ }^{10}$. Dann steht die Wahlbefugnis nicht unverrückbar fest, sondern kann in jedem Fall neu bestimmt werden, solange nur die Vorgaben des $\S 5$ Abs. 1 BVerfGG ("einer von dem einen, zwei von dem anderen Wahlorgan") im Ergebnis eingehalten werden. Ebenso gilt dann die Vorschrift zum Ruhen des Bundesrichteramtes für alle Bundesrichter. Diese Auffassung konnte sich zu recht im Ergebnis durchsetzen. Der Sinn der Bundesrichterklausel, den richterlichen Erfahrungsschatz einzubringen, wird durch sie vollständig gewahrt. Die formale Gruppenbetrachtung erfüllt demgegenüber spätestens seit Angleichung der Amtszeiten aller Verfassungsrichter keinen erkennbaren Zweck mehr. Das wiegt um so schwerer als ihre Konsequenzen dem Gesetzeswortlaut widersprechen: So bezieht sich § 70 DRiG unterschiedslos auf alle Bundesrichter. § 5 Abs. 1 BVerfGG hält die Verteilung der Wahlorgane gerade offen. Letzteres belegt auch der Gegenschluß aus Art. 3 S. 1 des Änderungsgesetzes von $1956^{11}$ sowie aus $\S 5$ Abs. 3 BVerfGG, die nur für Sonderfälle vorschreiben, daß Nachfolger und Vorgänger vom selben Wahlorgan zu wählen sind.

\section{FORMALER GANG DES WAHLVERFAHRENS}

Die Richter des Bundesverfassungsgerichts werden je zur Hälfte vom Bundestag und vom Bundesrat gewählt, Art. 94 Abs. 1 S. 2 GG. Die demokratische Legitimation des Bundesverfassungsgerichts, die zunächst funktionell und institutionell durch seine grundgesetzliche Konstituierung sowie in sachlich-inhaltlicher Hinsicht durch seine Anbindung an die Verfassung gesichert ist, wird durch diese kurze Legitimationskette in organisatorischpersoneller Hinsicht gestärkt ${ }^{12}$.

\subsection{Vorschlagslisten}

Als Hilfsmittel zur Bestimmung der Kandidaten für die Wahl zum Verfassungsrichter werden nach $\S 8$ BVerfGG beim Bundesminister der Justiz zwei kontinuierlich ergänzte Vorschlagslisten geführt. Eine dieser Listen enthält alle nach $\S 3$ Abs. 1 und 2 BVerfGG wählbaren Bundesrichter, die andere alle anderen wählbaren Personen, die von einem Berechtigten zur Wahl vorgeschlagen wurden. Vorschlagsberechtigt sind die Fraktionen des Bundestags, die Bundesregierung und die Landesregierungen. Die Listen werden den Präsidenten von Bundestag oder Bundesrat spätestens eine Woche vor der Wahl zugeleitet. Mangels Bindungswirkung ${ }^{13}$ ist ihre praktische Bedeutung gering. Kommen während der Beratungen der Wahlgremien weitere Kandidaten ins Spiel, so werden jedenfalls heute ${ }^{14}$ auch keine Nachtragslisten mehr erarbeitet. Die Listen werden im Justizministerium als Verwaltungsinterna behandelt und stehen der Öffentlichkeit nicht zur Einsicht zur Verfügung ${ }^{15}$.

\subsection{Die Wahl durch den Bundestag}

Der Bundestag wählt die Verfassungsrichter nicht direkt, sondern hat seine Befugnis in $\S 6$ BVerfGG auf einen eigens für diesen Zweck geschaffenen Wahlausschuß delegiert. Seine zwölf Mitglieder werden vom Plenum nach den Regeln der Verhältniswahl gewählt, wobei jede Fraktion eine Vorschlagsliste abgeben kann. Gemeinsame Listen mehrerer Fraktionen sind zulässig. § 6 Abs. 2 BVerfGG geht allerdings davon aus, daß mehrere Listen bestehen, also nicht etwa eine Einheitsliste aller Fraktionen aufgestellt wird ${ }^{16}$. In der Praxis stellen gegenwärtig die Regierungs- und Oppositionsfraktionen je eine gemeinsame Liste auf ${ }^{17}$. Abgestimmt wird nach der parlamentarischen Praxis, trotz fehlender gesetzlicher Vorschrift i.S.v. § 49 GOBT, mit verdeckten Stimmzetteln ${ }^{18}$. Jeder Abgeordnete hat eine Stimme, die er einer der für inn unveränderlichen Listen geben kann; Panaschieren oder Kumulieren ist nicht möglich. Die Anzahl der Mitglieder pro Liste wird sodann nach dem d'Hondtschen Höchstzahlverfahren 
errechnet ${ }^{19}$. Die Vorschlagsliste behält auch nach der Wahl Bedeutung. Scheidet ein Mitglied durch Tod, Mandatsbeendigung oder Verzicht endgültig aus oder ist es vorübergehend verhindert, so findet keine Nachwahl statt, sondern rückt der Listennachfolger ein, § 6 Abs. 2 S. 5 BVerfGG. Die Wahlmänner werden für die Dauer der Legislaturperiode gewählt und können nicht abberufen werden; sie unterliegen keinen Weisungen und sind zur Verschwiegenheit verpflichtet ${ }^{20}$. Dem Gremium kommt daher ein hohes Maß an Unabhängigkeit vom politischen Tagesgeschehen $\mathrm{zu}^{21}$.

Auf den Wahlausschuß finden die Regeln für Ausschüsse nach §§ 54ff. GO-BT Anwendung, soweit sich aus dem BVerfGG nichts abweichendes herleiten läßt. Er tagt nach $\S$ 69 Abs. 1 GO-BT und im Hinblick auf den Zweck der Verschwiegenheitspflicht des $\S 6$ Abs. 4 BVerfGG nichtöffentlich. Das nach dem Lebensalter älteste Mitglied ist verpflichtet, die Mitglieder unverzüglich unter Einhaltung einer Frist von einer Woche zur Durchführung der Wahl zu laden, § 6 Abs. 3 BVerfGG. Die Unverzüglichkeit richtet sich nach den in § 5 Abs. 2, 3 BVerfGG genannten Wahlzeitpunkten. Allerdings sollte hier nicht auf den spätestmöglichen ${ }^{22}$, sondern auf den frühestmöglichen Wahltermin abgestellt werden. Anderenfalls würde rechtstechnisch gerade für den Regelfall der Wahl nach Ablauf der Amtszeit des Vorgängers, für den $\S 5$ Abs. 2 1. Alt. BVerfGG nur den frühestmöglichen Zeitpunkt regelt, die Unverzüglichkeitspflicht ins Leere greifen. Die damit geförderte, möglichst frühe Wahl ist gerade im Hinblick auf die immer wieder auftretenden Verzögerungen ${ }^{23}$ auch rechtspolitisch sinnvoll. Zwar ist der immer wieder betonte zeitliche Entscheidungsspielraum des Ältesten praktisch wichtig, doch kann er seine Wirkung ebensogut im Rahmen der Beurteilung der Unverzüglichkeit selbst, d.h. des schuldhaften Zögerns (vgl. § 121 Abs. 1 S. 1 BGB), entfalten. Ohnehin kommt der Unverzüglichkeitsregel eher eine Appell- und Leitlinienwirkung zu, da ein Versto $ß$ nicht etwa die Richterwahl ungültig macht ${ }^{24}$. Die Ladungsfrist kann hingegen auch aufgrund ihrer Schutzfunktion für die Ausschußmitglieder nur mit deren Zustimmung unterschritten werden. Eine frühere Ladung ist möglich und für vorbereitende Sitzungen auch üblich. Die Wahlsitzung als solche wird fortgesetzt, bis alle Richter gewählt sind. Allerdings sind schon aus praktischen Gründen Vertagungen und Unterbrechungen möglich.

Der Wahlausschuß wählt die Bundesverfassungsrichter mit einer Mehrheit von mindestens 8 von 12 Stimmen. Die numerische Festlegung in $\S 6$ Abs. 5 BVerfGG setzt inzident die weniger strengen Regeln über die Beschlußfähigkeit nach § 67 GO BT außer Kraft: Es müssen mindestens 8 Mitglieder anwesend sein. Die 2/3-Mehrheit stellt einen Kompromiß dar. Einerseits sollen Regierung und Opposition zu einer Einigung gezwungen werden, so daß nicht etwa die jeweilige Regierungsmehrheit allein das Gericht besetzen und so politisch beeinflussen $\mathrm{kann}^{25}$. Andererseits darf die Wahl nicht durch ein zu strenges Mehrheitserfordernis behindert werden. Entsprechend wurde die ursprünglich vorgesehen 3/4Mehrheit schon 1956 durch die jetzige Regelung ersetzt, nachdem zuvor im Zusammenhang mit dem Kampf um den Wehrbeitrag zwei Jahre lang keine Einigung über eine Richternachfolge erzielt worden war ${ }^{26}$.

Das entscheidende Merkmal der Verfassungsrichterwahl durch den Bundestag wird allerdings in § 6 Abs. 5 BVerfGG nur am Rande erwähnt: Obwohl Art. 94 Abs. 1 S. 2 GG eine Wahl durch den Bundestag vorsieht, ist die Entscheidung des Wahlausschusses endgültig. Der Bundestag hat also sein Wahlrecht abschließend und ohne nachträgliche Korrekturmöglichkeit auf einen Ausschuß delegiert. Das Parlament behält nicht einmal eine staatsnotarielle oder auch nur symbolische Funktion: Wer bei einem Wahlvorgang im Ausschuß die erforderliche Stimmenzahl auf sich vereinigt, ist damit zum Richter gewählt. Das ist um so bemerkenswerter, als der Bundestag zugleich die Unabhängigkeit des Wahlausschusses mit starken rechtlichen Sicherungen versehen hat. Das Parlament hat sich damit, von der allgemeinen politischen Einflußnahme abgesehen, so vollständig wie nur möglich selbst aus dem Prozeß der Richterwahl ausgeschlossen.

\subsection{Die Wahl durch den Bundesrat}

Der Bundesrat wählt die Verfassungsrichter ohne Zwischenschaltung eines Ausschusses, aber ebenfalls mit 2/3-Mehrheit. Mangels weiterer Regelungen in § 7 BVerfGG findet für die Einzelheiten das Grundgesetz und die Geschäftsordnung des Bundesrats 


\section{DIE WAHL DER RICHTER AM DEUTSCHEN BUNDESVERFASSUNGSGERICHT ZWISCHEN RECHTLICHER THEORIE UND POLITISCHER PRAXIS}

Anwendung. Im Unterschied zum Bundestag findet die Wahl also öffentlich durch Handaufheben statt, Art. 52 Abs. 3 S. 3 GG, § 29 Abs. 1 S. 1 GO-BR. Die Stimmen jedes Landes können nur einheitlich abgegeben werden, Art. 51 Abs. 3 S. 2 GG. Aufgrund der geforderten 2/3-Mehrheit besteht in Abweichung von $\S 28$ GO-BR auch ein Quorum von 2/3 der Stimmen des Bundesrats.

In der Praxis wird die Wahl allerdings durch ein Kommission vorbereitet, in die alle Bundesländer je einen Vertreter, meist den Justizminister, entsenden. Sie ist es auch, die sich mit den Vorschlagslisten des Bundesjustizministers befaßt. Die Kommission erarbeitet in der Regel eine einzige Beschlußempfehlung. Das Plenum des Bundesrats nimmt diese Empfehlung regelmäßig ohne Debatte $a^{27}$.

\subsection{Der ergänzende Wahlvorschlag durch das Bundesverfassungsgericht}

Dieselben negativen Erfahrungen mit einer politisch bedingten Blockierung des Wahlverfahrens, die zur Herabsetzung der 3/4-Mehrheit auf 2/3 geführt hat, veranlaßten den Gesetzgeber auch, für den Fall von Verzögerungen zusätzliche Wahlvorschläge durch das Bundesverfassungsgericht vorzusehen, $\S 7 \mathrm{a} \mathrm{BverfGG}^{28}$. Kommt innerhalb von zwei Monaten nach Ablauf der Amtszeit oder nach Ausscheiden eines Richters keine Wahl im Verfahren nach $\S \S 6$ oder 7 BVerfGG zustande ${ }^{29}$, so hat im einen Fall der Älteste des Wahlausschusses, im anderen Fall der Präsident des Bundesrats oder sein Stellvertreter unverzüglich das Bundesverfassungsgericht zur Abgabe eines Wahlvorschlags aufzufordern.

\section{a. Verfahren im Plenum}

Der Vorschlag wird vom Plenum des Bundesverfassungsgerichts mit einfacher Mehrheit beschlossen. Geht es um die Wahl eines einzelnen Verfassungsrichters, ist eine Dreierliste einzureichen, anderenfalls eine Liste mit doppelt so vielen Kandidaten wie Richter zu wählen sind. Das Verfahren innerhalb des Bundesverfassungsgerichts richtet sich nach $\S \S$ 57-59 GO-BVerfG. Vorschlagsberechtigt innerhalb des Gerichts ist jeder Richter. Anders als in allen übrigen Varianten der Richterwahl ist der Vorschlag hier sogar zu begründen, $\S 57 \mathrm{~S} .2$ GO-BVerfG. Nach Aussprache wird allerdings geheim abgestimmt. Zur Beschlußfähigkeit ist die Anwesenheit von 2/3 der Mitglieder jedes Senats erforderlich, §§ 16 Abs. 2, 7a Abs. 2 S. 3 BVerfGG.

Das Bundesverfassungsgericht hat ein komplexes Verfahren entwickelt, um unter Wahrung der Beteiligungsrechte jedes Verfassungsrichters in angemessener Zeit eine Vorschlagsliste aufstellen zu können. Der Detailreichtum der internen Verfahrensreglung läßt darauf schließen, daß das Bundesverfassungsgericht der Erarbeitung eines Vorschlags nicht nur größere Bedeutung zumißt, sondern auch mit einer kontroversen und schwierigen Entscheidungsfindung rechnet.

Zunächst wird eine alphabetische Liste aller Kandidaten aufgestellt und ein erster Wahlgang durchgeführt, bei dem jeder Richter soviel Stimmen hat, wie dem Wahlausschuß Personen vorzuschlagen sind. Schon in diesem ersten Wahlgang sind diejenigen gewählt, die die Mehrheit der abgegebenen Stimmen auf sich vereinen, und zwar in der Reihenfolge der Stimmenzahl. Diese Regelung des $\S 58$ Abs. 2 GO-BVerfG macht allerdings nur Sinn, wenn Stimmen nicht kumuliert werden dürfen und unter der Mehrheit die absolute Mehrheit der erreichbaren Stimmen, also - wenn alle Verfassungsrichter ihre Stimmen abgeben - 9 oder mehr verstanden wird. Anderenfalls griffe entweder - bei absoluter Mehrheit ohne Kumulationsverbot - die Regelung zur Reihenfolge ins Leere, da immer nur eine Person die absolute Mehrheit erreichen könnte; oder es wäre - bei relativer Mehrheit mit oder ohne Kumulation - immer die erforderliche Anzahl von Kandidaten schon im ersten Wahlgang gewählt, womit die Regeln über weitere Wahlgänge überflüssig wären. Ungeregelt bleibt damit nur der Fall, daß ein Richter nur einen Teil seiner Stimmen abgibt, da dann nicht feststellbar ist, wieviele Stimmen als abgegeben gelten sollen. Werden im ersten Wahlgang nicht genügend oder gar keine Kandidaten gewählt, so finden anschließend weitere Wahlgänge in einem k.o.-Verfahren statt. Dabei schreibt jeder Richter pro Wahlgang einen Namen auf den 
Stimmzettel. Sobald ein Kandidat die Mehrheit der abgegebenen Stimmen erreicht, ist er gewählt. Dabei scheidet in jedem Wahlgang derjenige Kandidat aus, der im Wahlgang zuvor die wenigsten Stimmen erhalten hat ${ }^{30}$.

Führen diese Wahlgänge nicht zum Erfolg, so findet regelmäßig zwei Wochen später ein zweiter Wahltermin statt, $\S 59$ Abs. 1 S. 2 GO-BVerfG. Im zweiten Wahltermin wiederholt sich grundsätzlich das vollständige Verfahren des ersten Termins, einschließlich der verschieden gestalteten ersten und weiteren Wahlgänge. Zum zweiten Wahltermin können sowohl alte als auch neue Kandidaten benannt werden, wobei die Vorschlagsfrist sich auf drei Tage verkürzt. Von diesen Grundsätzen kann allerdings in zweierlei Hinsicht abgewichen werden. Erstens kann im zweiten Wahltermin durch Beschluß des Plenums auf den ersten (Listen-)Wahlgang verzichtet und sogleich mit den weiteren (Einzel-)Wahlgängen begonnen werden. Zweitens kann der zweite Wahltermin statt nach zwei Wochen auch sofort im Anschluß an den fehlgeschlagenen ersten Wahltermin stattfinden. Das setzt voraus, daß alle anwesenden Richter zustimmen und noch in der Sitzung Kandidaten für die neue Wahl vorgeschlagen werden. Handelt es sich dabei ausschließlich um Kandidaten, die bereits früher benannt waren, so bedarf es nur der Zustimmung von 2/3 der anwesenden Richter.

\section{b. Bedeutung des Wahlvorschlags}

Der Wahlvorschlag des Bundesverfassungsgerichts ist zunächst genau das: ein Vorschlag. Der Wahlausschuß kann jederzeit eine andere Person zum Verfassungsrichter wählen, § 7a Abs. 4 BVerfGG. Eine rechtliche Bindungswirkung kommt dem Vorschlag also nicht zu. Dies hat Beobachter immer wieder dazu veranlaßt, inm keine nennenswerte Wirkung zuzusprechen $^{31}$. Die Annahme eines Vorschlags des Bundesverfassungsgerichts müsse dem Wahlkörper als Eingeständnis des eigenen Unvermögens oder Versagens erscheinen und auch in den politischen Parteien und Länderregierungen auf Widerstand stoßen. Auch setze das Gericht seine überparteiliche Autorität aufs Spiel, wenn es in die tagespolitische Personaldiskussion eingreife ${ }^{32}$.

Tatsächlich begann die Praxis des § 7a BVerfGG mit einem Fehlschlag. 1961 wählte der Bundesrat keinen der vom Bundesverfassungsgericht vorgeschlagenen Kandidaten ${ }^{33}$. Auch das Bundesverfassungsgericht selbst schätzte diesen Beginn offenbar als wenig erfolgversprechend ein und reagierte über Jahre mit großer Zurückhaltung, nahm also jede Gelegenheit wahr, einen Vorschlag gar nicht erst abzugeben ${ }^{34}$. Bei der Nachfolge von Erwin Stein 1971 allerdings gab das Gericht erneut eine Vorschlagsliste ab. Ihr war trotz der politisch sehr umstrittenen Situation voller Erfolg beschieden; der Bundesrat wählte den Erstplatzierten ${ }^{35}$. Der Fall schien Schule zu machen. Bei der umstrittenen Nachfolge von Willi Geiger $1977^{36}$ gab die SPD schon vorab zu erkennen, daß sie zur Streitentscheidung einem Vorschlag des Bundesverfassungsgerichts folgen würde. So geschah es dann auch. In der Folge gab das Bundesverfassungsgericht seine Zurückhaltung endgültig auf. Als 1981 bei der Nachfolge von Martin Hirsch der Älteste des Wahlausschusses das Bundesverfassungsgericht erst mit zwei Monaten Verspätung zur Abgabe eines Vorschlags aufforderte und ihm überdies mitteilte, daß der Vorschlag jetzt nicht mehr berücksichtigt werden könne, weil man sich bereits auf einen Kandidaten geeinigt habe, verzichtete das Gericht keineswegs auf einen Vorschlag, sondern lieferte im Gegenteil eine Dreierliste, auf der sich gerade keiner der von den Parteien bevorzugten Kandidaten - auch nicht der kolportierte Favorit - wiederfand. Dieses mutige, aber taktisch nicht ungefährliche Einfordern des gebotenen Interorganrespekts ${ }^{37}$ hatte im Ergebnis Erfolg: Nicht nur vertagte der Wahlausschuß nach Eingang des Vorschlags die Verfassungsrichterwahl, er wählte auch später den auf der Liste Drittplatzierten und gerade nicht einen der zuvor favorisierten Kandidaten. Das Gericht hatte seine Position damit untermauert ${ }^{38}$. Aber auch die Wahlgremien hatten ihre Lektion gelernt und vermieden bei den Verfassungsrichterwahlen 1983 die Aufforderung an das Bundesverfassungsgericht, soweit die Wahl eines bestimmten Kandidaten ohnehin unmittelbar bevorstand ${ }^{39}$. Der dahinterstehende Gedanke, daß vom Zweck des $\S 7$ a BVerfGG her ein Vorschlag des Bundesverfassungsgerichts nicht notwendig ist, wenn eine rechtzeitige Wahl allein wegen technischer Schwierigkeit unterbleibt, leuchtet $\mathrm{ein}^{40} .1987 \mathrm{kam}$ es zu einem weiteren Vorschlag, dem das Wahlorgan jedoch nicht folgte ${ }^{41}$. Für die Folgezeit ist eine Analyse kaum noch möglich. Denn aus dem nicht immer konfliktlosen Miteinander von Wahlgremien und 


\section{DIE WAHL DER RICHTER AM DEUTSCHEN BUNDESVERFASSUNGSGERICHT ZWISCHEN RECHTLICHER THEORIE UND POLITISCHER PRAXIS}

Bundesverfassungsgericht ist dem Anschein nach eine weitere Konsequenz gezogen worden: Die Wahlvorschläge des Plenums werden spätestens seit Ende der 80'er Jahre vom Bundesverfassungsgericht als nichtöffentlich behandelt, insofern kein Akteneinsichtsrecht gewährt und auch nicht mitgeteilt, zu welchen genauen Wahlvorgängen überhaupt eine Vorschlag ergangen ist. Zwischen 1987 und 2004 kam es aber auch nur 1993 zu einer weiteren einschlägigen Beratung im Plenum ${ }^{42}$. Dabei konnte das Gericht sich mit seinem Vorschlag nicht durchsetzen, wollte dies aber nach Ansicht von Beobachtern im Hinblick auf eine politisierte Personaldiskussion auch nicht ${ }^{43}$. Immerhin wurde eine der Vorgeschlagenen auf die nächste freiwerdende Stelle gewählt.

Insgesamt hat sich § 7a BVerfGG als ein vielschichtiges Instrument erwiesen, dessen Erfolgsbilanz stark schwankt. Seine rechtstechnisch geringe verbindliche Wirkung verhinderte nicht seine potentielle, gelegentlich unter Beweis gestellte Zugkraft, die dem Bundesverfassungsgericht einen nicht geringen Einfluß bei streitigen Verfassungsrichterwahlen einräumt. Das Bundesverfassungsgericht hat dabei seine Überparteilichkeit gewahrt, die politischen Realitäten aber akzeptiert ${ }^{44}$ und gleichzeitig vornehmlich Personen vorgeschlagen, deren herausragende Eigenschaften mehr auf fachlichem als auf politischem Gebiet lagen. Der Balanceakt zwischen Einfluß und Politisierung war damit gelungen. Trotz oder gerade wegen des zeitweiligen Erfolgs spielte das Vorschlagsrecht aber in den vergangenen 20 Jahren keine große praktische Rolle mehr. Die zukünftige Entwicklung bleibt offen.

\section{DIE REALITÄT DES WAHLVERFAHRENS}

\subsection{Parteienproporz}

Die Realität des Wahlverfahrens wird durch die rechtlichen Vorgaben nur unvollkommen beschrieben und entscheidend von anderen Faktoren und Gepflogenheiten mitgeprägt. Zentrales Merkmal des Wahlverfahrens ist zunächst der Parteienproporz. Die 2/3Mehrheit verhindert die einseitige Besetzung freigewordener Richterstellen durch die jeweilige Regierungskoalition und soll einen Zwang zur Einigung schaffen. Die Parteien haben sich aber nicht darauf eingelassen, bei jeder Besetzung erneut in eine Grundlagendiskussion einzutreten. Sie haben stattdessen den effizienteren Weg einer Vorabfestlegung der partei- und machtpolitischen Verteilung gewählt, bei der dauerhaft feststeht, wem das Vorschlagsrecht für einen freiwerdenden Posten zusteht. Nach der in ihrer heutigen Ausprägung seit 1976 bestehenden Regelung steht der CDU/CSU sowie der SPD innerhalb jedes Senats das Vorschlagsrecht für jeweils 4 Richter zu. Drei dieser vier Stellen werden jeweils mit einem Parteigänger besetzt, die vierte mit einem neutralen Richter. Weiterhin treten die beiden großen Parteien für das ganze Bundesverfassungsgericht je einen ihrer 6 parteipolitisch zuzuordnenden Richter intern dem jeweils kleineren Partner ab. Diese über Jahre für die FDP praktizierte Lösung wird nunmehr auch auf Bündnis 90/Die Grünen angewandt ${ }^{45}$. Die in der Anfangszeit des Gerichts nicht gänzlich unberechtigte, auch heute immer wieder einmal erwähnte Unterscheidung zwischen dem "roten" Ersten Senat und dem "schwarzen" Zweiten Senat ${ }^{46}$ kann somit seit geraumer Zeit als Beschreibung der Modalitäten der Richterwahl nicht mehr aufrechterhalten werden.

Die Vereinbarung unter den Parteien ist allgemein bekannt, wurde aber niemals formal beschlossen oder gar niedergeschrieben. Als bloßer modus vivendi weist sie allerdings erstaunliche Wirksamkeit und Stabilität auf. Selbst bei hochumstrittenen Richterwahlen wurde sie als Grundlage der Zusammenarbeit nie ernsthaft in Frage gestellt. Dabei führt sie zu einem Grad an Formalisierung, wie er etwa der rechtsverbindlichen Regelung zu den Bundesrichterstellen am Bundesverfassungsgericht ${ }^{47}$ fehlt: Es steht im Grundsatz für jede einzelne Stelle fest, ob sie neutral oder parteigebunden ist, welcher Partei sie zusteht und wem daher das Vorschlagsrecht für den Nachfolger zukommt. Auch ist nicht nur jedem kundigen Beobachter, sondern jedem Richter des Bundesverfassungsgerichts bewußt, in welche Gruppe er fällt, so daß etwa ein als neutral gewählter Richter diesen neutralen Status auch intern stets im Blick haben wird.

\subsection{Parteigebundene und neutrale Richter}


Die Unterscheidung zwischen Parteigängern und neutralen Richtern ist dabei allerdings nur im Hinblick auf die bekannten Modalitäten des Wahlverfahrens zu referieren, unterliegt aber größten Bedenken, wenn aus ihr weitere Konsequenzen abgeleitet werden sollen. Einerseits relativiert sich die Unterscheidung stark, wenn bedacht wird, daß auch die neutralen Richter jeweils klar von einer der beiden großen Parteien ${ }^{48}$ vorgeschlagen werden und so parteipolitisch zuordenbar sind ${ }^{49}$. Doch wird bei der Wahl der neutralen Richter das volle Einverständnis des politischen Gegners gesucht, während bei den parteigebundenen Richtern die Gegenseite nur zurückhaltend Einwendungen geltend macht ${ }^{50}$. Dennoch werden beispielsweise die Sozialdemokraten für eine neutrale Stelle kaum eine Person vorschlagen, die durch ihre prägnant konservative Haltung aufgefallen ist, und umgekehrt. Weit wichtiger noch aber kann die Bezeichnung als "neutraler Richter" implizieren, daß die übrigen Richter nicht neutral wären. Damit aber würde an den Wurzeln der Verfassungsgerichtsbarkeit und des in sie gesetzten Vertrauens gerührt ${ }^{51}$. Auch die parteigebundenen Richter sind keine "Parteisoldaten". Daß die Richter sich bei ihrem Abstimmungsverhalten am Wohl ihrer Partei, an der Parteilinie oder an Parteitagsbeschlüssen orientierten, gehört - erfreulicherweise - nicht zu den Vorwürfen, die dem Verfassungsgericht gegenüber ernsthaft erhoben würden. Im Gegenteil fallen immer wieder Richter auf, die in prominenten Fällen gänzlich anders entscheiden, als ihre Parteizugehörigkeit vermuten ließe. Zwar läßt sich nicht bestreiten, daß die Regelung des Wahlrechts theoretisch eine starke parteipolitische Orientierung der Richter ermöglicht. Doch darf in einer zahlenmäßig so kleinen Gruppe der Stil des Hauses, der gelebte und für jeden Neuberufenen prägende Amtsethos der Richter nicht unterschätzt werden ${ }^{52}$. Mit diesem Selbstverständnis der Bundesverfassungsrichter aber ist ein parteipolitisches Vorgehen unvereinbar und wäre im Gericht nicht akzeptabel.

\subsection{Vereinheitlichung unter den Wahlgremien}

Verfassungsrichter werden also nicht, wie das Bundesverfassungsgerichtsgesetz nahezulegen scheint, in der Diskussion des unabhängigen Wahlausschusses nominiert, sondern vorab im Rahmen einer informalen Einigung der Parteien untereinander. Damit wird zugleich der rechtlich bedeutsame Unterschied zwischen den vom Bundestag und den vom Bundesrat zu wählenden Richtern in seiner praktischen Bedeutung beseitigt und durch die Unterscheidung zwischen von der SPD oder der CDU/CSU zu nominierenden Kandidaten ersetzt. Die Parteien einigen sich auf die Kandidaten völlig unabhängig davon, ob diese vom Wahlausschuß oder vom Bundesrat $z u$ wählen sind. Das wird besonders bei sog. Paketlösungen deutlich, bei denen mehrere Richter, deren Wahl in nicht allzugroßem Abstand voneinander ansteht, gleichzeitig und in Abhängigkeit voneinander bestimmt werden. Welches Wahlgremium dafür verantwortlich ist, spielt bei diesen Paketen keine Rolle. Diese Einebnung führt sogar dazu, daß in der kritischen Diskussion Parteienproporz und Wahlausschuß regelmäßig zusammen behandelt werden, die Wahl durch den Bundesrat hingegen allenfalls am Rande erwähnt und keineswegs als Gegenmodell zum Wahlauschuß wahrgenommen wird.

\subsection{Obleute}

Die Verlagerung der faktischen Wahlentscheidung aus den offiziell zuständigen Gremien heraus geht noch einen Schritt weiter. Nicht nur bestehen informelle, von den Bundestagsfraktionen beschickte Arbeitsgruppen ${ }^{53}$, über die kaum etwas näheres bekannt ist. Wichtiger noch liegt ein Großteil der Entscheidungsgewalt im Ergebnis bei zwei Obleuten. Dabei handelt es sich um fachlich wie persönlich in Juristenkreisen bewanderte Personen, die je einer der beiden großen Parteien angehören und die Personalentscheidung auch langfristig vorbereiten. Sie sind für die jeweilige Gegenseite der zentrale Ansprechpartner. Entsprechende Obleute gibt es auch auf Seiten des Bundesrats ${ }^{54}$. Wie diese Obleute bestimmt werden und ob es dazu überhaupt ein erkennbares Verfahren gibt, ist nicht bekannt. Zumindest einschlägig interessierten Kreisen bekannt ist hingegen in der Regel die Identität der Obleute ${ }^{55}$.

\subsection{Nichtöffentlichkeit}




\section{DIE WAHL DER RICHTER AM DEUTSCHEN BUNDESVERFASSUNGSGERICHT ZWISCHEN RECHTLICHER THEORIE UND POLITISCHER PRAXIS}

Kennzeichnend für den ganzen Wahlprozeß ist damit vor allem eine ausgeprägte Nichtöffentlichkeit. Im Wahlausschußverfahren ist sie durch § 6 Abs. 4 BVerfGG gesetzlich festgelegt. Im übrigen aber führt die faktische Auslagerung der Entscheidungen in die Parteien und hier auf die Obleute dazu, daß der Bürger und oft sogar die Parlamentarier ${ }^{56}$ nicht wissen können, wie es zu der Entscheidung für den einen und gegen den anderen Kandidaten kam, ja wer überhaupt als Kandidat in der engeren Wahl stand. Das gilt auch für das Verfahren im Bundesrat, bei dem zwar theoretisch eine öffentliche Diskussion möglich wäre, das aber in der Praxis durch einen Beschluß ohne Aussprache gekennzeichnet ist. Bis auf gelegentliche Indiskretionen aus Parteikreisen ist das gesamte Verfahren auf ein Höchstmaß an Undurchsichtigkeit angelegt.

\section{KRITIK DES WAHLVERFAHRENS: VERFASSUNGSRECHTLICHE UND VERFASSUNGSPOLITISCHE ASPEKTE}

Entsprechend groß ist die Kritik am Verfahren, das manchem "undemokratischer als die Papstkür ${ }^{157}$ erscheint, von vielen Stimmen in der Staatsrechtslehre vehement verurteilt und nur selten in Schutz genommen wird. Die Kritik hat eine lange Tradition ${ }^{58}$ und ist heute in ihrer Vielzahl kaum noch überschaubar ${ }^{59}$. Dabei werden die verschiedensten Reformvorschläge gemacht (1). Anstelle eines Generalangriffs auf das gegenwärtige Verfahren sollte allerdings zwischen den verschiedenen Hauptkritikpunkten unterschieden werden, also dem Parteiproporz (2), der mangelnden Öffentlichkeit des Verfahrens (3) und der Zwischenschaltung von Wahlmännern durch den Bundestag (4). Gemeinsamer Hintergrund dieser Punkte ist wiederum das jeweilige Vorverständnis zum Bild von Verfassungsgerichtsbarkeit und der Rolle der Verfassungsrichter (5).

\subsection{Reformvorschläge}

An Vorschlägen zur Reform der Verfassungsrichterwahl herrscht kein Mangel. Sie betreffen typischerweise drei Kategorien, nämlich Öffentlichkeit, Gremien und Wahlmodus. Innerhalb und zwischen ihnen sind die verschiedensten Kombinationen möglich.

\section{Öffentliche Anhörung}

Zur Gewährleistung größerer Öffentlichkeit wird ein Gedanke immer wieder ins Spiel gebracht: die öffentliche Anhörung. Dabei sollen nach US-amerikanischem Vorbild die näher in Betracht kommenden Kandidaten vor einem Wahl- oder Vorbereitungsgremium in öffentlicher Sitzung persönlich erscheinen und zwecks Ermittlung ihrer Eignung Rede und Antwort stehen. So soll das Verfahren transparenter und der Vorrang sachlicher wie fachlicher Gesichtspunkte bei der Auswahl gesichert werden ${ }^{60}$. Zum Teil wird aber auch betont, daß die in der Anhörung zu stellenden Fragen in Grenzen auch persönlicher Natur sein könnten und dürften, da auch solche Gesichtspunkte die Entscheidung faktisch beeinflußten ${ }^{61}$.

\section{(2) Wahlgremien}

Für die Wahlgremien wird gelegentlich eine Vereinheitlichung vorgeschlagen, bei der alle Richter durch den Wahlausschuß des Bundestages gewählt und dann durch den Bundesrat bestätigt würden ${ }^{62}$. Andere fordern eine direkte Wahl durch den Bundestag selbst anstelle des Wahlausschusses. Dies war Gegenstand einer Reihe im Kern übereinstimmender Gesetzesentwürfe, die von den Grünen in der 11. bis 13. Wahlperiode erfolglos eingebracht wurden $^{63}$. Vielfach wird auch die Einbeziehung eines Beirats befürwortet. Bekanntester Ausdruck dieses Bestrebens war eine Gesetzesinitiative Mitte der 50'er Jahre, nach der bei erfolgloser Wahl binnen zwei Monaten ein Beirat einzuberufen war, der aus zwei Bundesgerichtspräsidenten, zwei Professoren und drei Landesverfassungsgerichtspräsidenten bestehen sollte. Dieser Beirat sollte mit einfacher Mehrheit eine kurze Vorschlagsliste empfehlen, aus der das Wahlorgan dann mit einfacher Mehrheit einen Verfassungsrichter 
wählen konnte ${ }^{64}$. Die Befürworter sahen darin eine Möglichkeit der Entpolitisierung der Wahl, die damalige SPD-Opposition hingegen den Versuch, ihre Einflußmöglichkeiten im Zusammenspiel eines tendenziell eher konservativen Beirats und der Wahl mit einfacher Mehrheit auszuschalten. Sie ließ den Entwurf daher scheitern ${ }^{65}$. Der Gedanke wurde in der einen oder anderen Form immer wieder aufgegriffen, etwa in Form eines Bundestag und Bundesrat gemeinsamen, paritätisch beschickten Vorbereitungsausschusses ${ }^{66}$. Auch der Gedanke einer stärkeren Einbeziehung von juristischen Fachleuten fand immer wieder Anklang. So wurde etwa ein juristischer Fachbeirat mit Vertretern aller Berufsgruppen, aus denen sich Verfassungsrichter typischerweise rekrutieren, vorgeschlagen, der angehört werden müßte, aber nur unverbindliche Empfehlungen abgeben könnte ${ }^{67}$. Auch ein weitergehender Einfluß einer "Elite der unabhängigen hohen Richterschaft" innerhalb eines Richterwahlausschusses wurde schon früh gefordert ${ }^{68}$. Schließlich wird immer wieder einmal auch dem Bundespräsidenten eine besondere Rolle zugedacht, sei es als stimmberechtigter Vorsitzender eines Wahlausschusses ${ }^{69}$, als Berufungsberechtigter für einen Beirat ${ }^{70}$ oder als Reservemacht für den Fall, daß eine Einigung der sonst berufenen Gremien nicht rechtzeitig zustandekommt $^{71}$. Damit wird versucht, die besondere Rolle des Bundespräsidenten als Integrations- und Identifikationsfigur, sein besonderes Ansehen und seine Neutralität nutzbar zu machen und so auch dem Bundesverfassungsgericht aufzuprägen.

\section{(3) Wahlmodus}

Schließlich werden diverse Vorschläge zur Modifikation des Wahlmodus gemacht. Ein früher Regierungsvorschlag, die 2/3-Mehrheit zugunsten einer einfachen Mehrheit aufzugeben, hat allerdings kaum Widerhall gefunden ${ }^{72}$. Ein besonders überraschender Gedanke bestand darin, statt einer Wahl schlicht das Los entscheiden zu lassen. Dabei soll zwecks "Entpolitisierung, Rejustifizierung, Reprofessionalisierung, Entprofilierung" das Gericht nur noch aus obersten Bundesrichtern bestehen, unter denen bei jeder Berufung das Los entschiede $^{73}$. Der Gedanke erinnert an antike Formen der Ämterbesetzung ${ }^{74}$. Wiederum andere sind der Ansicht, daß der von einem Vorbereitungsgremium ausgearbeitete Wahlvorschlag für das Wahlgremium bindend sein sollte, das also auf die inm vorgeschlagenen Kandidaten beschränkt wäre ${ }^{75}$. Damit soll die im Vorbereitungsgremium durchzuführende Anhörung gesichert werden ${ }^{76}$. Schließlich wurde zur Förderung von Öffentlichkeit und Objektivität des Verfahrens immer wieder einmal eine Begründungspflicht für den Wahlvorschlag gefordert ${ }^{77}$.

\subsection{Kritik am Parteienproporz}

\section{a. Ungebührlicher Parteieneinfluß?}

Die Kritik am bestimmenden Einfluß der Parteien speist sich zunächst aus einem diffusen Gefühl der Unanständigkeit, das sich in negativ besetzten Begriffen wie "Kuhhandel", "Erbhöfe" oder "Machtpositionen" niederschlägt ${ }^{78}$. Dahinter steht konkreter die Furcht vor einer Politisierung des Verfassungsgerichts. Richter, die jeweils von einer vorgegebenen Partei bestimmt werden und überwiegend sogar Parteimitglieder sind, stehen mit dem Bild einer über der Parteipolitik stehenden, nur dem Recht verpflichteten Gerichtsbarkeit nicht in Einklang. Vor allem beim Bürger kann so das Bild eines parteipolitisch dominierten Gerichts hervorgerufen werden. Verfassungsgerichtsbarkeit könnte zur Fortsetzung der Politik in einem anderen Forum degenerieren. Das gilt um so mehr, als nach Ansicht mancher die parteigebundene Besetzungsmethode weniger hervorragende Staatsrechtler als vielmehr eben Parteigänger zu Richtern erhebt ${ }^{79}$. Schließlich wird dem Parteiproporz vorgehalten, er verhindere die demokratische Bestimmung der Richter. Sie würden nicht mehr, wie von Art. 94 GG vorgesehen, von Bundestag und Bundesrat gewählt sondern von diffusen Parteizirkeln hinter den Kulissen ausgekungelt ${ }^{80}$. Die gewählten Abgeordneten verlören so ihren notwendigen Einfluß $\beta^{81}$. 


\section{DIE WAHL DER RICHTER AM DEUTSCHEN BUNDESVERFASSUNGSGERICHT ZWISCHEN RECHTLICHER THEORIE UND POLITISCHER PRAXIS}

\section{b. Parteiendemokratie des Grundgesetzes}

Die Auffassung stellt sich zunächst als Teil der gängigen Kritik am "Parteienstaat" dar $^{82}$. Die Parteien haben demnach einen übergroßen Einfluß sowohl im gesellschaftlichen als auch im staatlichen Bereich. Alle wichtigen Posten in gesellschaftlichen Institutionen, aber auch zahlreiche staatliche Ämter seien ihrem Machtstreben ausgesetzt und würden von ihnen besetzt, die dort gefällten Entscheidungen damit von ihnen mitbestimmt. Parteibuchwirtschaft und Ämterpatronage bestimmten das Bild der Republik ${ }^{83}$.

So angebracht solche Bedenken politisch auch gelegentlich sein mögen, darf doch aus verfassungsrechtlicher Sicht damit nicht die Legitimität der zentralen Rolle der Parteien in der parlamentarischen Demokratie übersehen werden. Die Demokratie des Grundgesetzes hängt nicht dem idealisierten Bild eines harmonischen Volksganzen nach, das einen gemeinsamen, einheitlichen Willen bildet und inn über das Parlament zu Geltung bringt. Eine solche Vorstellung der Identität von Herrschern und Beherrschten eignet sich eher als Kampfbegriff gegen die Demokratie, indem ein utopischer Urzustand zum Maßstab erhoben wird, demgegenüber die Realität nur als zynisch erscheinen kann ${ }^{84}$. Unter dem Grundgesetz sind stattdessen die unterschiedlichen, oft unvereinbaren Strömungen und Vorstellungen in der Gesellschaft und ihren Untergruppen als gegeben hinzunehmen. Um sie in ihrer Disparität zu bündeln und zu integrieren, bedarf es aber gerade der Parteien; Parteien sind deshalb für die parlamentarische Demokratie unabdingbar. Mit Art. 21 GG ist diese Funktion auch verfassungsrechtlich verankert: Die Parteien spielen bei der staatlichen Entscheidungsfindung eine entscheidende Rolle ${ }^{85}$. Der Parteienstaat, oder besser: die Parteiendemokratie ${ }^{86}$, steht nicht im Gegensatz zur "wahren" Demokratie, sondern ist im parlamentarischen System ihr unabdingbarer Ausdruck. Ein generelles Mißtrauen gegenüber dem Einfluß der Parteien ist damit unabgebracht.

Das gesagt, ist die verfassungsmäßige Rolle der Parteien aber auch nicht unbeschränkt. Nicht nur sind sie an der Willensbildung des Volkes nur beteiligt, ersetzen diese also nicht; wichtiger noch sind sie - im Gegensatz zu Leibholz' klassischer Vorstellung vom Parteienstaat - nicht in den staatlichen Herrschaftsapparat eingefügt ${ }^{87}$ oder werden gar mit den Staatsorganen auf eine Stufe gestellt und mit innen verflochten ${ }^{88}$. Die sich daraus ergebenden verfassungsrechtlichen Grenzen des Parteieneinflusses sind für und innerhalb jeder Staatsgewalt eigenständig zu bestimmen. So wird zu recht davon ausgegangen, daß den Parteien gerade im Bereich der Justiz enge Grenzen gezogen sind, die sich aus der für diese konstitutiven richterlichen Unabhängigkeit, Art. $97 \mathrm{GG}$, ergeben ${ }^{89}$. Für die Bundesverfassungsrichter besteht aber eine Besonderheit: Das Grundgesetz selbst ordnet ihre Wahl durch die Legislativorgane an. Gerade in den Legislativorganen aber macht sich der Einfluß der Parteien und ggf. ihrer Fachgremien oder Obleute am deutlichsten bemerkbar. Gerade parlamentarische Entscheidungen werden unabwendbar von Parteien und Parteipolitik mitbestimmt $^{90}$; dies ist der Kern der Anerkennung der Rolle der Parteien in der parlamentarischen Demokratie. Werden Entscheidungen somit Bundestag und Bundesrat selbst übertragen, so liegt darin zugleich eine Übertragung in den direkten Einflußbereich der Parteien ${ }^{91}$. Insofern ist die Parteiabhängigkeit der Verfassungsrichterwahl nicht nur nicht verfassungswidrig, sondern im Grundgesetz selbst angelegt.

\section{c. Parteinähe und Vorverständnis}

Damit mag allerdings zwar der bestimmende Einfluß der Parteien, aber noch nicht die personelle Besetzung der Stellen nach Parteibuch oder geistiger Nähe zur Partei legitimiert sein. In der Tat könnten die Parteien dazu übergehen, nur noch nach der Fachkompetenz auszuwählen. Doch wird bei aller Kritik am gegenwärtigen System die fachliche Kompetenz der von diesem System hervorgebrachten einzelnen Verfassungsrichter interessanterweise nie ernsthaft bezweifelt. Tatsächlich stellt gerade die 2/3-Mehrheit und der damit verbundene Zwang zur Einigung nicht nur sicher, daß allzu eindeutige Parteigänger wenig Aussicht auf Erfolg haben, sondern auch daß die fachliche Qualität aller Kandidaten hoch ist. Denn anderenfalls droht stets ein Eingreifen des politischen Gegners und damit die politisch blamable Situation, einen öffentlich als nicht hinreichend kompetent gebrandmarkten 
Kandidaten vorgeschlagen zu haben und damit gescheitert zu sein. Problematisch, aber letztlich kaum lösbar ist allein, daß eine ganze Reihe von hervorragenden Kandidaten gar nicht erst in Betracht kommen, weil sie den Parteien nicht genügend ins Auge gefallen sind.

Davon abgesehen ist hier, wie oft, die Vorstellung von der "reinen" Fachkompetenz irrig $^{92}$. Sie impliziert, daß sich die richtige Entscheidung allein oder jedenfalls in erster Linie aus dieser Fachkompetenz ergibt, daß sie also zumindest ganz überwiegend anhand rein objektiver, rechtswissenschaftlicher Kriterien zu ermitteln ist. Das bedeutet aber nichts anderes als die Vorstellung, daß es die objektiv richtige Entscheidung gibt, die nur noch mit der gehörigen Portion Fachwissen und Intelligenz gefunden werden muß. Dem ist aber nicht so. In der heutigen Methodenlehre herrscht in einem Punkt Einigkeit: Das juristische Urteil folgt in vielen Fällen gerade nicht rein logisch aus der Rechtsnorm in Verbindung mit den zugrundezulegenden empirischen Tatsachen ${ }^{93}$. Stattdessen fließen Werte und Wertungen in die rechtliche Entscheidungsfindung mit ein ${ }^{94}$. Werte und Wertungen sind allerdings ihrerseits nicht nur subjektive Motivationen oder rein rechtspolitisches Wünschen, sondern werden gerade durch die notwendige Entscheidungsbegründung nach außen gesteuert und gefiltert, so daß der Rechtsbindung des Art. 20 Abs. 3 GG Rechnung getragen wird ${ }^{95}$. Entsprechend berichten erfahrene Verfassungsrichter von einer "Brechung der außer- oder vorjuristischen Überzeugungen" in der verfassungsgerichtlichen Praxis ${ }^{96}$. In der täglichen Arbeit ist der Jurist es aber dennoch gewohnt, verschiedene Ergebnisse für vertretbar zu halten, also als rational auf dem Gesetz fußend zu akzeptieren ${ }^{97}$. Das gilt besonders ausgeprägt für die Verfassungsgerichtsbarkeit und hier für die Grundrechte und Staatsorganisationsprinzipien, die durch ein besonderes $\mathrm{Ma} B$ an sprachlicher Offenheit und Auslegungsbedürftigkeit gekennzeichnet $\operatorname{sind}^{98}$. Damit gewinnen gerade bei Verfassungsrichtern ethisch-politische Grundanschauungen und Wertungen, von denen sich kein Jurist freisprechen kann eine besondere Bedeutung, die schon bei den Beratungen im parlamentarischen Rat immer wieder betont wurde ${ }^{99}$. Ginge es wirklich nur um die fachkompetente Anwendung der Verfassung, so könnte zudem die sachlich-inhaltliche Legitimation ohne weiteres ausreichen und bedürfte es keiner - inhaltlich gerade nicht an bestimmten Maßstäben orientierten ${ }^{100}$ - Wahl direkt durch die gesetzgebenden Körperschaften.

Soll die Rechtsprechung des Gerichts nicht einseitig werden, ist es deshalb erforderlich, auch im Hinblick auf die weltanschaulichen Grundpositionen eine ausgeglichene Besetzung anzustreben. Die parteipolitischen Präferenzen können dabei durchaus als guter Anhaltspunkt gelten. Es geht also nicht um die genaue Parteilinie, sondern um Grundhaltung und verfassungsrechtliches Vorverständnis, die durch Parteizugehörigkeit oder Parteinähe indiziert werden ${ }^{101}$. Die Alternative, ausschließlich oder überwiegend parteipolitisch Neutrale in das Gericht zu berufen, ist nur eine Scheinlösung. Denn auch diese Neutralen haben ihr Vorverständnis - nur daß es oft weit weniger offensichtlich und daher weit weniger berechenbar ist. Das offizielle Parteimitglied als Richter ist insofern sogar das kleinere Problem. Gerade soweit also versucht wird, die Ausgewogenheit des Bundesverfassungsgerichts zu sichern, liegt im parteipolitischen Proporz nicht die Krankheit, sondern das verläßlichste - wenn auch manchem bitter erscheinende - Heilmittel.

Dabei hat der Proporz dem weiterhin hohen Ansehen des Gerichts ${ }^{102}$ keinen Abbruch getan und nicht den Eindruck parteipolitischer Entscheidungen hervorgerufen ${ }^{103}$. Für das notwendige Maß an Differenzierung und Eigenständigkeit der Positionen sorgt die intellektuelle Kapazität der Richter und spätestens das Gericht selbst. Urteils- und manchmal auch Gerichtsschelte gehört zwar durchaus zum Alltag. Doch kommt diese Schelte - wie schon vielfach beobachtet wurde - in schöner Regelmäßigkeit mal aus der einen, mal aus der anderen politischen Richtung, ganz abhängig davon, wessen politische Meinung sich in aktuellen Einzelfällen gerade nicht durchsetzen konnte ${ }^{104}$. Eben darin aber liegt der beste Beleg der fehlenden (partei-)politischen Voreingenommenheit der Richter und ihrer Entscheidungen. Etwas subtiler zeigt sich dies auch in den gelegentlich offengelegten, wechselnden Mehrheiten sowie in der Seltenheit von 4:4 Entscheidungen. Würde die hälftige Aufteilung beider Senate zwischen den beiden großen politischen Lagern tatsächlich zu einer entsprechenden Stimmenverteilung bei politisch wichtigen Entscheidungen führen, müßten die 4:4 Entscheidungen weit öfter auftreten. 


\section{DIE WAHL DER RICHTER AM DEUTSCHEN BUNDESVERFASSUNGSGERICHT ZWISCHEN RECHTLICHER THEORIE UND POLITISCHER PRAXIS}

Im Ergebnis sollte das stabile Verteilungssystem des Parteienproporzes nicht als Negativum, sondern im Gegenteil als ein markant positives Merkmal des Bundesverfassungsgerichts betrachtet werden.

\subsection{Kritik an der mangelnden Öffentlichkeit}

Der oft wiederholte Vorschlag, den Schleier der Geheimhaltung nach US-amerikanischem Vorbild durch eine öffentliche Anhörung zu lüften, erscheint auf den ersten Blick ideal. Die Anhörung würde die Kandidaten, ihren fachlichen Hintergrund, ihre Ansichten sowie ihre Persönlichkeit vorstellen und dabei in der Praxis aufgrund des zu erwartenden großen Interesses der Presse einer breiten Öffentlichkeit zugänglich machen. Zwar bliebe das Warum der eigentlichen Wahlentscheidung weiterhin im Herzen der Stimmberechtigten - seien es Wahlmänner oder nunmehr alle Bundestagsabgeordneten - verborgen, doch würde das gesamte Verfahren transparenter, jeder Bürger könnte sich ein eigenes Bild machen. Zugleich wären die Stimmberechtigten sich dieser Öffentlichkeit bewußt, so daß die oft kritisierte "Kungelei" kleiner (Partei-)Zirkel jedenfalls erheblich erschwert würde. Wenig, so scheint es zunächst, kann gegen einen solchen Vorschlag sprechen, der zudem endlich Kants transzendentaler Formel des öffentlichen Rechts Rechnung trüge: "Alle auf das Recht anderer Menschen bezogenen Handlungen, deren Maxime sich nicht mit der Publicität verträgt, sind unrecht." 105

\section{a. Vorbild USA?}

Das Problem steckt nicht im Grundgedanken der öffentlichen Anhörung, sondern in der damit unvermeidlich verbundenen Praxis und ihren Folgen. Gerade die Realität im Stammland dieses Vorschlags, den USA, kann nur als abschreckend bezeichnet werden ${ }^{106}$. Das gesamte Berufungsverfahren für Richter am Supreme Court und damit auch die Anhörung ist hochgradig politisiert. Im Rahmen der Anhörung werden die Kandidaten zu ihren früheren Veröffentlichungen und ggf. fachrichterlichen Entscheidungen im Detail ebenso befragt wie zu ihrer Meinung zu zentralen Fragen wie etwa der Abtreibung ${ }^{107}$ oder der Gleichberechtigung ${ }^{108}$. Mehr noch stehen die Richter auch mit ihren persönlichen Eigenschaften und ihrer persönlichen Integrität auf dem Prüfstand. Zwei radikale Fälle aus den vergangenen 20 Jahren lassen die möglichen Konsequenzen erkennen.

Extreme in fachlicher Hinsicht tauchten bei der Nominierung von Robert Bork auf. Er war Anhänger der verfassungstheoretischen Schule des original intent. Diese Schule will die verfassungsrechtlich garantierten Rechte und ihre Auslegung weitgehend nur am Willen der Gründungsväter festmachen - eine Auffassung, die bei einer über 200 Jahre alten Verfassung von vielen als problematisch angesehen, von ihren Anhängern als neutral und von ihren Gegnern als radikal konservativ betrachtet wird ${ }^{109}$. Der von Lobbygruppen mit 2 Millionen Dollar finanzierte Kampf gegen die Nominierung führte in der Anhörung zu einer Detailbefragung, mit der versucht wurde, Borks zukünftiges Abstimmungsverhalten im Supreme Court im Einzelnen vorherzusagen ${ }^{110}$. Kritiker erarbeiteten und verbreiteten ein sog. "Book of Bork", das seine Veröffentlichungen und Reden zusammenstellte. Bork versuchte im Rahmen der Anhörung auf alle Fragen ruhig, rein fachlich und auf hohem akademischen Niveau zu antworten. Gerade dieser Stil wurde jedoch kritisiert ${ }^{111}$. In einer vielzitierten Rede zur Nominierung Borks brachte Senator Kennedy inn in Verbindung mit Rassendiskriminierung, Abtreibungen durch Kurpfuscher, Zensur und Polizeistaat; er schloß mit dem Satz "No justice would be better than this injustice."112 Borks Behandlung wurde in der gespaltenen Literatur zum Teil als schändlich bezeichnet ${ }^{113}$. Verfassungsrichter wurde Bork nicht.

Zu einer anderen Art von Extrem kam es Anfang der 90'er Jahre bei der Nominierung von Clarence Thomas. Während des Anhörungsverfahrens erhob eine frühere Mitarbeiterin Vorwürfe, von Thomas (verbal) sexuell belästigt worden zu sein. Die Medien stürzten sich auf die vor laufender Kamera gemachten Anschuldigungen, sie wurden im Anhörungsverfahren debattiert und von Thomas vehement zurückgewiesen. Er selbst sah darin - in Anspielung auf seine schwarze Hautfarbe - "a high tech lynching of an uppity black man"114. Die Anhörung, die 
Thomas schließlich gewann, wurde plakativ als Schlachtfeld eines veritablen politischen Krieges beschrieben ${ }^{115}$.

\section{b. Politisierung, Personalisierung, Banalisierung}

Wenn auch in der deutschen Literatur weitgehend Einigkeit zu herrschen scheint, daß Anhörungen in diesem Stil und mit diesem Inhalt keinesfalls wünschenswert wären, wird doch betont, daß solche Auswüchse in Deutschland vermieden werden könnten. Dafür soll zunächst schon eine Definition der Anhörung sorgen ${ }^{116}$. So werden etwa zum Teil Fragen nach politischen und religiösen Grundüberzeugungen für legitim, Fragen aus Bereichen wie Familie oder sexuelle Orientierung hingegen für illegitim gehalten ${ }^{117}$. Andere wollen die Fragen auf beruflichen Werdegang, Zugehörigkeit zu bestimmten, insbesondere politisch oder weltanschaulich relevanten Gruppierungen sowie bisherige öffentliche Ämter beschränken ${ }^{118}$. Weiterhin wird darauf hingewiesen, daß die Anhörung in den USA vom politischen Kampf zwischen Präsident und Senat bestimmt sei. Ähnlichem Verhalten beuge in Deutschland der Einigungszwang durch die 2/3-Mehrheit vor ${ }^{119}$. Im übrigen könnten an Bewerber um ein so wichtiges Amt auch einige Anforderungen hinsichtlich der Offenlegung ihrer Verhältnisse gestellt werden ${ }^{120}$.

Tatsächlich besteht die Möglichkeit, daß eine Anhörung in Deutschland in ruhigen, sachlichen und angemessenen Bahnen abliefe und so einen positiven Zuwachs an Öffentlichkeit und gelebter Demokratie brächte. So gab es in früheren Zeiten auch in den USA einen sehr wohlwollenden Umgang mit den Kandidaten ${ }^{121}$. Mehr als eine Prognose ist insofern nicht möglich. Diese Prognose aber fällt grundlegend negativ aus. Die Wahl der Verfassungsrichter ist in Deutschland zwar kein Kampf zwischen zwei Staatsgewalten, aber ein Kampf zwischen den Parteien, die in einer - unweigerlich sehr medienwirksamen und dadurch geprägten - Anhörung eine Chance zur Profilierung hätten. Die Chancen einer Selbstbeschränkung in Bezug auf den Inhalt der Fragen wären dabei von der politischen Selbstdisziplin aller - ggf. auch sehr kleinen und randständigen - beteiligten Parteien abhängig. Gerade bei umstrittenen Kandidaten oder in allgemeinpolitisch angespannter Lage ist damit aber nach aller Erfahrung allenfalls beschränkt zu rechnen. Gerade die schon jetzt vertretenen, unterschiedlichen Positionen zum sinnvollen Inhalt einer solchen Beschränkung, etwa im Hinblick auf politische Grundüberzeugungen, zeigen die möglichen Debatten über eine Selbstbeschränkung und damit deren offene Grenzen auf. Gleichzeitig könnte bei einer zu engen Beschränkung etwa auf aktenkundige Tatsachen mit gutem Recht bezweifelt werden, welchen Sinn die Anhörung noch haben sollte ${ }^{122}$. Zweifelhaft ist zudem, wie - im Hinblick auf die angestrebte Versachlichung - die juristische Kompetenz oder allgemein fachliche Fragen aus dem Verfassungsrecht in einer öffentlichen Anhörung angemessen "abgefragt" oder sonst beurteilt werden sollen. Zwischen wenig interessanten Fragen nach Aktenlage und kaum möglicher Begutachtung rein fachlicher Fähigkeiten bleiben letztlich nur Fragen nach persönlichen und politischen Ansichten. Mit anderen Worten wird das Mehr an Öffentlichkeit mit einem Mehr an Politisierung und Ideologisierung erkauft.

Der Zwang zur Einigung könnte in wenig umstrittenen Fällen zur Mäßigung führen, doch darf selbst dann nicht übersehen werden, daß die Anhörung in Deutschland - gerade im Unterschied zu den USA - nicht der Bestätigung einer schon vorab gefällten Entscheidung für eine bestimmte Person, sondern im Gegenteil der Vorbereitung einer Auswahl unter mehreren Kandidaten dienen soll. Die Anhörung wäre also nicht mit den in anderen Bereichen üblichen Anhörungen von wissenschaftlichen Sachverständigen vergleichbar, sondern stellten schon konzeptionell eine Konkurrenzsituation zwischen den Befragten sowie zwischen den sie befürwortenden Gruppierungen dar, die eine Polarisierung in der Anhörung fördert.

Damit zusammenhängend besteht die Gefahr, daß öffentliche Anhörungen in zweierlei Hinsicht zu einer Verengung auf bestimmte Richterpersönlichkeiten führen. Zum einen könnten eine Reihe von geeigneten Kandidaten eine Bewerbung ablehnen, weil sie sich - aus nachvollziehbaren Gründen - einem solchen Prozeß nicht unterziehen wollen ${ }^{123}$. Das gilt um so mehr als - im Unterschied zu den USA ${ }^{124}$ - stets mehrere Kandidaten offiziell als Verlierer dastünden und als solche in Erinnerung blieben. Im gegenwärtigen Verfahren hingegen ist allenfalls kleinen Kreisen - und dann oft nicht mit Sicherheit - bekannt, gegen wen sich der 


\section{DIE WAHL DER RICHTER AM DEUTSCHEN BUNDESVERFASSUNGSGERICHT ZWISCHEN RECHTLICHER THEORIE UND POLITISCHER PRAXIS}

letztlich erfolgreiche Kandidat durchgesetzt hat. Zum anderen können in öffentlichen Anhörungen vor allem Personen einen guten Eindruck hinterlassen, die es verstehen, publikumswirksam aufzutreten, elegant zu formulieren, gewandt oder gar gewitzt zu antworten und insgesamt zu gefallen. Sie dürften zudem eher fähig und bereit sein, die Fallstricke und Gefahren einer öffentlichen Anhörung auf sich zu nehmen. Auch solche Persönlichkeiten braucht das Bundesverfassungsgericht, aber es wäre bedauerlich, wenn weniger öffentlichkeitswirksame, gefällige oder geschickte Juristen bei gleicher sonstiger Qualifikation weniger oder gar keine Chance hätten.

\subsection{Kritik an der Mediatisierung im Wahlausschußverfahren}

\section{a. Verfassungsrechtliche Fragestellung}

Die verfassungsrechtliche Problematik des Wahlausschußverfahrens liegt in der klaren Formulierung des Art. 94 Abs. 1 S. 2 GG, der dem Bundestag selbst das Wahlrecht gibt und im Gegensatz zu Art. 95 Abs. 2 GG gerade keinen Wahlausschuß erwähnt ${ }^{125}$. Die Argumente pro und contra variieren hier wenig ${ }^{126}$ : Aus Wortlaut und Systematik ist in beide Richtungen ein argumentum e contrario möglich, da einerseits die Bestellung wichtiger Ausschüsse im Grundgesetz zum Teil ausdrücklich geregelt ist (vgl. Art. 45, 95 Abs. 2 GG), andererseits auch eine gewünschte Unmittelbarkeit der Wahl ausdrücklich festgelegt wird (vgl. Art. 28 Abs. $1 \mathrm{~S}$. 2, 38 Abs. $1 \mathrm{~S}$. $1 \mathrm{GG}$ ). Daß darüber hinaus eine verfassungswidrige Praxis allein durch ihr jahrelanges Bestehen aufgrund der normativen Kraft des Faktischen verfassungsmäßig werden, ja eine Verfassungswandlung bewirken könnte ${ }^{127}$, wäre allenfalls als Entwicklung derogierenden Verfassungsgewohnheitsrechts denkbar, wofür es aber in Anbetracht der kontroversen Diskussion an der notwendigen opinio iuris fehlen würde ${ }^{128}$. Zwei weitere Argumente bedürfen hingegen näherer Betrachtung. Erstens wird die notwendige demokratische Legitimation des Bundesverfassungsgerichts zum Teil bezweifelt, da sie ihm aufgrund seiner besonderen Aufgaben direkt vom Bundestag vermittelt werden müsse, zum Teil aber bejaht, da sie auch bei Zwischenschaltung eines Ausschusses erhalten bleibe. Zweitens und damit zusammenhängend wird darauf hingewiesen, daß der Bundestag aufgrund seiner Geschäftsordnungsautonomie das Recht habe, Angelegenheiten zu delegieren. Dem wiederum werden mögliche Begrenzungen der Autonomie im Bereich zentraler Kompetenzen entgegengehalten, die nicht rein innere Organangelegenheiten beträfen ${ }^{129}$.

\section{b. Verfassungspolitische Fragestellung}

Verfassungspolitisch ist das Wahlausschußverfahren entgegen vielen Stimmen jedenfalls zu begrüßen. Es stellt sicher, daß im kleinen und fachkundigen Kreis offen und ohne Behinderung durch Erwägungen von Wahlkampf und Außendarstellung über die Wahlentscheidung diskutiert werden kann. Der Wahlausschuß teilt insofern die bekannte Vorzüge der Ausschußverfahren, wie sie insbesondere aus der Gesetzgebung bekannt sind ${ }^{130}$. Eine Verlagerung in das Plenum des Bundestages würde die Unvoreingenommenheit der Debatte verhindern. Wichtiger noch würde die angestrebte Offenlegung des Entscheidungsprozesses nicht erreicht. Die Vorstellung, die einzelnen Abgeordneten würden offen und im Dialog das pro und contra des einen und anderen Kandidaten erörtern und so schließlich vor den Augen aller - einschließlich der anwesenden Presse - in transparenter Weise zu einer Entscheidung kommen, ist ein Idealbild, das den parlamentarischen Realitäten und der bedeutenden, verfassungsmäßigen Rolle der Parteien nicht Rechnung trägt. Je nach Ausgestaltung der Diskussion können hier zudem die gegenüber einer öffentlichen Anhörung bestehenden Bedenken durchgreifen. In der Praxis würde bei Abschaffung des Wahlausschusses die Entscheidung in einem nunmehr informellen Gremium vorgefaßt und sodann vom Bundestag nur noch bestätigt. Aufgrund des Einigungszwangs hätten allenfalls noch kleine parlamentarische Gruppierungen, die sich im Entscheidungsprozeß nicht wiederfänden, ein Interesse an einer kontroversen Debatte. Der nicht mehr berechenbaren Vorentscheidung durch informelle Kreise ist aber die zumindest gewisse Steuerung durch einen 
formalen Wahlausschuß vorzuziehen. Der Wahlausschuß mag nicht die beste denkbare Lösung sein, er ist aber die beste mögliche.

\section{c. Vergleich mit dem Bundesratsverfahren}

Welches Legitimationsniveau für das Bundesverfassungsgericht verfassungsrechtlich erforderlich ist, ist konkret schwer zu bestimmen, da es um die Grenzfrage geht, ob die Zwischenschaltung eines weiteren Glieds in der Legitimationskette - des Wahlausschusses gerade noch oder gerade nicht mehr hinnehmbar ist. Eine wirklich eindeutige, überzeugende Lösung ist in solchen Situationen kaum zu finden, wie die nicht enden wollende Diskussion anschaulich belegt. Was fehlt, ist eine genauere grundgesetzliche Vorgabe, eine konkreterer Maßstab. Tatsächlich findet sich ein solcher Maßstab aber im Grundgesetz selbst, nämlich in Art. 94 Abs. 1 S. 2 GG, der die Wahl einer Hälfte der Bundesverfassungsrichter durch den Bundesrat anordnet. Der Bundesrat aber ist im Gegensatz zum Bundestag nicht direkt demokratisch gewählt, sondern über Landesparlamente und vor allem die Landesregierungen nur sehr mittelbar ${ }^{131}$. Das Grundgesetz selbst besteht also bei der Wahl von Verfassungsrichtern nicht auf einer unmittelbaren demokratischen Legitimation durch ein direkt vom Volk gewähltes Organ, sondern läßt auch eine weit indirektere Bestimmung ausreichen. Zwar gewinnt der Bundesrat durch seine Absicherung im Text des Grundgesetzes eine zusätzliche funktionelle und institutionelle Legitimation, die dem Wahlausschuß so fehlt. Doch kann diese eher schwache Legitimationsform die personelle Legitimation nicht ersetzen ${ }^{132}$, ist m.a.W. das Gesamtlegitimationsniveau der Wahl durch den Bundesrat nicht so hoch, daß ihr nur die direkte Wahl durch das Bundestagsplenum und nicht durch einen von diesem gewählten Ausschuß zu entsprechen vermöchte.

\section{d. Beteiligung aller Abgeordneten}

Damit verbleibt die Frage, ob die Delegation der Wahlentscheidung an einen Ausschuß von der Organisationsgewalt des Bundestages erfaßt ist. Grundsätzlich ist entsprechend parlamentarischer Tradition die Delegation auf Ausschüsse, die soweit wie möglich ein verkleinertes Abbild des Parlaments darstellen, nicht zu beanstanden ${ }^{133}$. Jedoch wird beim Wahlausschuß, im Unterschied zu sonstigen Ausschüssen, nicht nur die Diskussion und Vorbereitung sondern die Entscheidung selbst delegiert. Die übliche Ausschußarbeit verkürzt aber gerade deshalb nicht das jedem Abgeordneten aus seinem Status zukommende Stimmrecht nach Art. 38 Abs. 1 S. 2 GG, weil es weiterbesteht und bei der Beratung im Plenum geltend gemacht werden $\operatorname{kann}^{134}$. So hat das Parlament bei der Gestaltung seiner Geschäftsordnung zwar einen erheblichen Gestaltungsspielraum. Rechtlicher Kontrolle unterliegt jedoch, ob dabei das Prinzip der Beteiligung aller Abgeordneten an den Aufgaben des Parlaments gewahrt bleibt ${ }^{135}$. Zu den Verfassungsgrundsätzen, die der Bundestag bei der Regelung seiner Geschäftsordnungsangelegenheiten zu beachten hat, gehört vor allem das Prinzip der repräsentativen Demokratie ${ }^{136}$. Das bedeutet zwar nicht, daß die Abgeordneten das Volk nur im Plenum des Bundestages repräsentieren könnten ${ }^{137}$. Bei der positiven Beurteilung der Ausschußarbeit wird jedoch vorausgesetzt, daß die endgültige Beschlußfassung über ein parlamentarisches Vorhaben dem Plenum vorbehalten bleibt ${ }^{138}$. Die Geschäftsordnungsautonomie erlaubt es dem Bundestag demnach vor dem Hintergrund der demokratischen (Stimm-)Rechte aller Abgeordneten nicht, inm von der Verfassung zugewiesene Entscheidungen abschließend an einen Ausschuß zu delegieren. Ebensowenig, wie etwa der Bundestag die endgültige Abstimmung über ein Gesetz einem Ausschuß übertragen könnte, kann er die endgültige Wahl der Verfassungsrichter übertragen. Dem läßt sich auch nicht etwa die Arbeitsfähigkeit des Parlaments als Verfassungsrechtsgut entgegenhalten ${ }^{139}$, da die Arbeitsfähigkeit des Parlaments durch die Endabstimmung im Plenum in keiner Weise beeinträchtigt, sondern sogar in ihrem Kerngehalt gewahrt wird.

Der verfassungsrechtliche Befund ist also ebenso zwiespältig wie überraschend: Das Wahlausschußverfahren ist rechtspolitisch sinnvoll und auch im Hinblick auf die demokratische Legitimation nicht zu beanstanden. Dennoch ist es verfassungswidrig, da die endgültige Abstimmung über eine vom Grundgesetz dem Parlament übertragene Frage im Hinblick auf die Abgeordnetenrechte nicht in einen Ausschuß delegiert werden darf. Durch die genaue 


\section{DIE WAHL DER RICHTER AM DEUTSCHEN BUNDESVERFASSUNGSGERICHT ZWISCHEN RECHTLICHER THEORIE UND POLITISCHER PRAXIS}

Abgrenzung des Problems ist aber auch die Lösung ohne größeren Aufwand möglich: Das bisherige Verfahren kann uneingeschränkt beibehalten werden. Die Abstimmung im Wahlausschuß darf allerdings nur zu einem unverbindlichen Vorschlag an das Plenum führen, das darüber - ohne Aussprache - abstimmen muß. Als positiver Nebeneffekt einer solchen Regelung würden durch die entsprechende Änderung des § 6 BVerfGG auch die immer wieder geäußerten Bedenken im Hinblick auf die demokratische Legitimation verschwinden, ohne die Vorteile des gegenwärtigen Verfahrens aufzugeben.

\section{Hintergrund des Streits: Das Bild der Verfassungsgerichtsbarkeit}

a. Verfassungsgerichtsbarkeit zwischen politischer und rechtlicher Entscheidung

Wie die Verfassungsrichterwahl abschließend bewertet wird, hängt letztlich von der schwer faßbaren Frage ab, wo die Verfassungsgerichtsbarkeit im Spektrum zwischen politischer und rechtlicher Entscheidung verortet wird. Wer in der Verfassungsgerichtsbarkeit eine primär politische und wenig von Rechtstext und Rechtsdogmatik angeleitete Entscheidungsform sieht, wird ihre sachlich-inhaltliche demokratische Legitimation gering einschätzen, daher eine Wahl fordern, wie sie auch sonst zur Besetzung der politischen Staatsleitung erforderlich ist, entsprechend also auf einer direkten Wahl durch den Bundestag, öffentlicher Diskussion der Kandidaten und Offenlegung auch ihrer politischen und weltanschaulichen Ansichten ebenso wie ihrer persönlichen Qualitäten bestehen. Die Parteizugehörigkeit wird tendenziell als in der parlamentarischen Demokratie natürlich erscheinen, solange die letztendliche Bestimmung nicht in den Arkanbezirken der Parteienmacht verschwindet ${ }^{140}$. Wer hingegen das Bundesverfassungsgericht - bei aller Ablehnung eines mechanistischen, positivistischen Rechtsverständnisses und Anerkennung des oft politischen Entscheidungsgegenstandes - als Rechtsprechung ansieht, die Probleme allein anhand eines Rechtsmaßstabes entscheidet ${ }^{141}$, wird tendenziell - wie hier - ihre sachlich-inhaltliche Legitimation stärker einschätzen, die Bedeutung einer möglichst direkten Wahl, die auch bei anderen Richtern nicht üblich ist, weniger betonen, und die Bedeutung der (partei-)politischen und weltanschaulichen Ausgewogenheit im Gericht nur aus pragmatischen Gründen anerkennen, um einerseits eine möglichst von fachlichen Kriterien bestimmte Entscheidung zu gewährleisten, andererseits die gerade im Verfassungsrecht real bestehenden, unterschiedlichen politisch-gesellschaftlichen Grundeinstellungen gegeneinander auszubalancieren.

\section{b. Die rechtskulturelle Verwurzelung der Frage}

Eine allgemeinverbindliche Antwort auf die Frage, was Verfassungsgerichtsbarkeit ist, gibt es allerdings nicht, ja kann es in der heutigen Welt nicht geben. Ähnlich wie beim verwandten Thema der richtigen oder angewendeten Methodenlehre ${ }^{142}$ ist die Verfassungsgerichtsbarkeit so politisch oder so rechtsprechend, wie sie in der jeweiligen Rechtskultur praktiziert wird. "Die" Verfassungsgerichtsbarkeit, also ein Abstraktum, das aus theoretischen Gründen auf eine bestimmte Art und Weise einzuordnen wäre, existiert nicht. So verwundert es nicht, daß Stimmen, die die Anbindung des Verfassungsgerichts an die Verfassung als verbindlichen Rechtsmaßstab bezweifeln und Verfassungsgerichtsbarkeit als ein politisches Verfahren sehen wollen ${ }^{143}$ sich oft aus US-amerikanischen Quellen speisen oder an diese anlehnen. Denn tatsächlich ist die Steuerungskraft des Verfassungstextes und einer Verfassungsrechtsdogmatik in den USA insgesamt sehr viel geringer als in Deutschland. In Deutschland hingegen ist - von außen und aus der Vogelperspektive betrachtet - ein im USSinne sehr viel "formalistischerer"143 Rechtssprechungsstil auch im Verfassungsrecht verbreitet $^{144}$. Die steuernde Kraft des Verfassungstextes und der dazu erarbeiteten Dogmatik wird hier gerade von Verfassungsrichtern nicht bezweifelt ${ }^{145}$.

\section{c. Politisches Verständnis als selbsterfüllende Prophezeiung}

Verfassungsgerichtsbarkeit ist also ein politisches Geschäft, wenn die Verfassungsrichter an die politische Bestimmung ihres Amtes glauben und sich entsprechend 
verhalten. Damit ist aber nicht etwa eine Einbahnstraße im Sinne eines schlichten Empirismus beschrieben. Denn nicht nur bestimmt die Realität die Theorie, sondern auch die Theorie die Realität: Wenn die Ansicht Oberhand gewinnt, die das Verfassungsgericht als politisches Organ ansieht, dann kann, im Wechselspiel von öffentlicher Meinung ${ }^{146}$, Staatsrechtslehre ${ }^{147}$ und Verfassungsgericht, diese Ansicht nach und nach die Realität bestimmen. Wird, wie hier, eine solche Entwicklung u.a. im Hinblick auf die Berechenbarkeit der Rechtsprechung und die Wahrung des deutschen Rechtsstils als unerwünscht betrachtet, dann sollte das gegenwärtige Wahlsystem auch deshalb erhalten bleiben, weil es das unpolitische Selbstverständnis der Verfassungsrichter fördert. Würde die Besetzung der vollen Wucht des öffentlichen politischen Kampfes ausgesetzt, die unvermeidliche parteipolitische Einfärbung der Kandidatensuche zum Gegenstand allgemeiner Debatte, die Notwendigkeit einer parteipolitischen und damit weltanschaulich ausgewogenen Besetzung immer aufs neue beleuchtet und hinterfragt, so läge darin eine Politisierung von Amt und Gericht, die im Wege eine selbsterfüllenden Prophezeiung das Gericht selbst verändern könnte. Der heilsame Mythos des unpolitischen Gerichts jedoch bedarf um seiner stetigen Verwirklichung Willen der Pflege.

\section{NOTES}

* deutscher Rechtswissenschaftler und derzeit Lehrstuhlinhaber an der Ernst-Moritz-ArndtUniversität Greifswald.

1. Vgl. etwa Klaus Kröger, Richterwahl, in: Christian Starck (Hg.), Bundesverfassungsgericht und Grundgesetz, 1976, S. 76 (84) (für Vorschlagslisten); Theodor Maunz, in: BVerfGG Komm., § 3 Rn. 2 (für Wahl); Hans Lechner/Rüdiger Zuck, BVerfGG, ${ }^{4} 1996$, § 3 Rn. 10 (für Ernennung).

S. u. Rn. 14.

Zum Streit bei Entstehung des Gesetzes vgl. Wilhelm Karl Geck, Wahl und Amtsrecht der Bundesverfassungsrichter, 1986, S. $15 f$.

Vgl. zur Entwicklung Geck (N 3), S. 17f.

Vgl. Wiltraut Rupp-von Brünneck, Darf das Bundesverfassungsgericht an den Gesetzgeber appellieren?, in: FS für Gebhard Müller, 1970, S. 355 (378).

Ausdrücklich für seine Einbeziehung schon BVerfGE 1, 208 (259).

BVerfGE 65, 152 (157).

Zu einem Fall der Nichtbeachtung der Sollbestimmung vgl. Henning Frank, Die Mitwirkung des BVerfG an den Richterwahlen, in: FS Hans Joachim Faller, 1984, S. 37 (45f.). Dabei ging es interessanterweise um den Wunschnachfolger desjenigen Verfassungsrichters, der sich zuvor für die Verwandlung in eine zwingende Norm und die Verlängerung der Frist auf 5 Jahre eingesetzt hatte, vgl. Willi Geiger, Zur Reform des Bundesverfassungsgesetzes, in: FS Hans Nawiasky, 1956, S. $211 \mathrm{ff}$.

Zur Statistik vgl. Richard Ley, Die Wahl der Mitglieder des Bundesverfassungsgerichts, in: ZParl 1991, S. 420 (443ff.).

Zum ganzen so Willi Geiger, Über den Umgang mit dem Recht bei der Besetzung des

Bundesverfassungsgerichts, in: EuGRZ 1983, S. 397 (398ff.).

So Benno Erhard, Über allzu schnelle Kritik an Verfassungsorganen bei der Wahl von

Bundesverfassungsrichtern, in: EuGRZ 1983, S. 473 (474ff.); BVerfGE 65, 152 (155ff.).

Vgl. dazu BVerfGE 65, 152 (156).

Zu den Formen demokratischer Legitimation vgl. zusammenfassend BVerfGE 93, 37

(66f.); weitere Nachweise bei Uwe Kischel, Die Begründung, 2003, S. 107f. m.w.N.; mit Bezug auf Richterwahlen Ernst-Wolfgang Böckenförde, Verfassungsfragen der Richterwahl, ${ }^{2} 1998$, S. $71 \mathrm{ff}$.

Kritisch insofern allein Geiger (N 10), S.398.

Von einer anderen Praxis berichtet noch Kröger (N 1), S. 88.

Auskunft des Bundesjustizministeriums an den Verfasser vom 29. März 2004.

Ebenso Franz Klein, in: BVerfGG Komm., § 6 Rn. 5.

Vgl. für die 15. Wahlperiode BT-Drucks $15 / 140$ und 15/141 v. 03.12.2002.

Vgl. etwa Diemut Majer, in: Dieter Umbach/Thomas Clemens (Hg.), BVerfGG, Mitarbeiterkommentar und Handbuch, 1992, § 6 Rn. 12; Klein (N 17), § 6 Rn. 6.

In der 15. Wahlperiode ergaben sich so je 5 Sitze für SPD und CDU/CSU sowie je ein Sitz für FDP und Bündnis 90/Die Grünen, vgl. zum Wahlergebnis Plenarprotokoll 15/14 vom 5. Dezember 2002, S. 1041 A, B.

Vgl. Majer (N 19) § 6 Rn. 13; Klein (N 17) § 6 Rn. 8f. 


\section{DIE WAHL DER RICHTER AM DEUTSCHEN BUNDESVERFASSUNGSGERICHT ZWISCHEN RECHTLICHER THEORIE UND POLITISCHER PRAXIS}

21. Daß allerdings meist prominente Abgeordnete entsandt würden, so noch Wilhelm Karl Geck, Wahl und Status der Bundesverfassungsrichter, in: HStR II, ${ }^{2} 1998$ ('1987), § 55 Rn. 7, läßt sich jedenfalls gegenwärtig nicht feststellen.

So offenbar Klein (N 17) § 6 Rn. 10.

S. u. Rn. $52 f f$.

Zur Rechtsfolge ebenso Klein (N 17) § 6 Rn. 10.

Zur entgegengesetzten Praxis in den USA vgl. etwa Henry Julian Abraham, Justices, presidents and senators, Lanham 1999, S. 3ff.; Jörg Zätzsch, Richterliche Unabhängigkeit und Richterauswahl in den USA und Deutschland, 2000, S. $115 \mathrm{ff}$.

Vgl. im Einzelnen Geck (N 3), S. 25ff.

Vgl. zum Ganzen etwa Christoph Gusy, Das Parlament als Wahlorgan, Gesetzgeber und Prozeßpartei im Verhältnis zum BVerfG, in: Schneider/Zeh (Hg.), Parlamentsrecht und Parlamentspraxis in der Bundesrepublik Deutschland, 1989, § 60 Rn. 14; Majer (N 19) $\S 6$ Rn. $5 \mathrm{ff}$.

28. Zur Entstehungsgeschichte vgl. Geck (N 3), S. 25ff.

29. Die Vorschrift betrifft auch Wahlen durch den Bundesrat, § 7a Abs. 3 BVerfGG; daß $\S$ 7a Abs. 1, 3 BVerfGG nur $\S 6$ und nicht $\S 7$ BVerfGG nennen, dürfte auf einem Redaktionsversehen beruhen.

Vor 1989 schieden sogar jeweils zwei Kandidaten aus. Diese Regelung wurde jedoch verändert, um die Liste der verfügbaren Kandidaten nicht zu schnell zu leeren; vgl. KarlGeorg Zierlein, in: Umbach/Clemens (Hg.), BVerfGG, Mitarbeiterkommentar und Handbuch, 1992, § 7a Rn. 27.

Vgl. Geiger (N 10), S. 398; Kröger (N 1), S. $89 f$.

Kröger (N 1), S. $89 f$.

Vgl. Henning Frank, Die ,neutralen' Richter des BVerfG, in: FS für Wolfgang Zeidler, 1987, S. 163 (167).

Einzelheiten bei Frank (N 8), S. 40ff.

Vgl. Frank (N 8), S. 43f.

Vgl. Frank (N 8), S. 45f.; vgl. dazu auch schon o. Rn. 3 Fn. 8.

Zum Interorganrespekt vgl. umfassend Ralph Alexander Lorz, Interorganrespekt im Verfassungsrecht, 2001.

Vgl. Rolf Lamprecht, Kungelei hinter den Kulissen, in: DRiZ 1986, S. 314; Frank (N 8), S. 46f., zur dezenten Bekundung von Genugtuung durch den damaligen Präsidenten des Bundesverfassungsgerichts vgl. ibd., S. 47.

Vgl. Erhard ( $N$ 11), S. 474, der Älteste des Wahlausschusses teilte dem Bundesverfassungsgericht 9 Tage nach Ablauf der Zwei-Monats-Frist mit, daß die Wahl 14 Tage später stattfinden würde; Frank (N 8), S. 48f.; darauf bezieht sich auch die Kritik von Geiger (N 10), S. 398.

Vgl. Erhard (N 11), S. 474

Zierlein (N 31), § 7a Rn. 36.

Mündliche und schriftliche Auskunft der Direktorin beim Bundesverfassungsgericht an den Verfasser vom 16. März 2004; der Verfasser bedankt sich für das freundliche Entgegenkommen; da der Wechsel von Thomas Dieterich an die Spitze des Bundesarbeitsgerichts erst zum Jahreswechsel 1993/94 bekannt wurde - vgl. "Auftritt der Restvernunft", SZ vom 5. Jan. 1994 - kann sich dieser Vorgang nur auf die Nachfolge von Ernst Gottfried Mahrenholz beziehen; zur parallelen Nichtöffentlichkeit der Vorschlagslisten nach § 8 BVerfGG vgl. o. Rn. 5.

Vgl. näher Heribert Prantl, Der Kampf um Karlsruhe, SZ vom 27. Okt. 1993; den Akten des BVerfG selbst allerdings läßt sich zu einem Ergebnis der Beratung nichts entnehmen, vgl. schriftliche Auskunft der Direktorin beim Bundesverfassungsgericht an den Verfasser vom 16. März 2004.

So hat es selbst bei der offenen Konfrontation mit dem Wahlausschuß 1981 von vornherein nur Kandidaten vorgeschlagen, die der insofern nach den politischen Gepflogenheiten - dazu u. Rn. 17 - vorschlagsberechtigten SPD angehörten; vgl. Lamprecht (N 39), S. 314.

Vgl. zum ganzen etwa Ernst-Wolfgang Böckenförde, Verfassungsgerichtsbarkeit: Strukturfragen, Organisation, Legitimation, in: NJW 1999, S. 9 (16 Fn. 31); Rolf Lamprecht, Parteisoldaten(innen) in Robe, in: NJW 1999, S. 2011 (2011f.); zur historischen Entwicklung informativ Frank (N 34), S. 163ff. und Kröger (N 1), S. $96 f f$.

Bei jeweils 12 Mitgliedern pro Senat standen anfänglich im 1. Senat 6 SPD-Mitglieder 2 Richtern der CDU, einem der FDP und 3 parteilosen gegenüber, im 2. Senat hingegen war die Verteilung 3 SPD, 1 CDU, 1 CSU, 7 Parteilose, vgl. dazu näher Frank (N 34), S. 166; 
das Bundesverfassungsgericht selbst wies diese Terminologie offiziell stets zurück, vgl. Kröger (N 1), S. 95 m.w.N.

Dazu o. Rn. 3.

Gemeinhin kommt dabei das Vorschlagsrecht für eine der neutralen Richterstellen der

CDU/CSU der bayerischen Staatskanzlei, d.h. der CSU zu, vgl. Frank (N 34), S. 170.

Deutlich etwa Lamprecht (N 46), S. 2011.

Vgl. Böckenförde (N 46), S. 16 Fn. 31.

Vgl. dazu noch u. Rn. 27, 33f., 49, 72 f.

Josef Isensee, BVerfG - quo vadis?, in: JZ 1996, S. 1085 (1093) spricht von einem

"Wechsel der Loyalität mit dem Wechsel des Amtes".

Vgl. BT-Drucks. 13/2088 v. 27.7.1995, S. 3.

Vgl. Friedrich Karl Fromme, Verfassungsrichterwahl, in: NJW 2000, S. 2977 (2978);

Helmut Kerscher, Selbst die Papstwahl ist demokratischer, SZ vom 5. Dez. 1998.

Namensnennung etwa bei Stephan Detjen, Kur-Kartell oder: Kungeln für Karlsruhe, in:

ZRP 2001, S. 93 (93); Rolf Lamprecht, 'Bis zur Verachtung', in: NJW 1995, S. 2531

(2532); Fromme (N 55), S. 2978.

Vgl. nur den Bericht von Detjen (N 56), S. 93.

Volker Neumann, Undemokratischer als die Papstkür?, Frankfurter Rundschau v. 22. Nov. 1999, S. 10.

Vgl. bereits Richard Thoma, Rechtsgutachten betreffend die Stellung des Bundesverfassungsgerichts vom 15. März 1953, in: JöR 1957, 161 (186ff.), z.T. allerdings mit einer heute nicht mehr gängigen Stoßrichtung (Bestimmung von Verfassungsrichtern durch das Parlament als "seltsame Paradoxis", ibd., 187); zur kontroversen Diskussion bereits bei der Beratung des BVerfGG vgl. Sybille Koch, Die Wahl der Richter des BVerfG, in: ZRP 1996, 41 (42) m.w.N.

Für einen ersten Überblick vgl. die Listen bei Wolfram Höfling/Thomas Roth, Ungesetzliche Bundesverfassungsrichter?, in: DÖV 1997, S. 66 (67 Fn. 4); Thomas Trautwein, Bestellung und Ablehnung von Bundesverfassungsrichtern, 1994, S. 18 Fn. 1; für die ältere Literatur vgl. Werner Billing, Das Problem der Richterwahl zum BVerfG, 1969, S. 229 Fn. 1.

Vgl. etwa Lamprecht (N 56), S. 2353; Ulrich K. Preuß, Die Wahl der Mitglieder des BVerfG als verfassungsrechtliches und -politisches Problem, in: ZRP 1988, S. 389 (394f.);

Kröger (N 1), S. 99; entsprechend frühere Gesetzentwürfe der Grünen, vgl. BT-Drucks. 11/73 vom 20.3.1987; BT-Drucks. 12/5375 vom 5.7.1993; BT-Drucks. 13/1626 v. 2.6.1995; BT-Drucks. 13/2088, v. 27.7.95.

Vgl. Preuß (N 61), S. 395.

Vgl. Kröger ( $N$ 1), S. 98f.; Wunsch nach möglichst wenig auseinanderfallenden

Wahlverfahren auch bei Klaus Stern, Gedanken zum Wahlverfahren für Bundesverfassungsrichter, in: GS für Karl Wilhelm Geck, 1989, S. 885 (895).

BT-Drucks. 11/73 vom 20.3.1987; BT-Drucks. 12/5375 vom 5.7.1993; BT-Drucks. $13 / 1626$ v. 2.6.1995; BT-Drucks. 13/2088 v. 27.7.95; seit der Regierungsbeteiligung der Grünen ab der 14. Wahlperiode wurden solche Gesetzentwürfe allerdings nicht mehr eingebracht.

Zu den Einzelheiten und zur damaligen Diskussion ausführlich Richard Häußler, Der Konflikt zwischen BVerfG und politischer Führung, 1994, S. 44ff., 188ff.

Stattdessen kam es zur Schaffung des heutigen § 7a BVerfGG, vgl. o. Rn. 11ff.

Vgl. Billing (N 60), S. 301ff.; Kröger (N 1), S. 99.

Vgl. Billing (N 60), S. 307ff.

Vgl. Thoma (N 59), S. 188; zur Verfassungswidrigkeit der Kooptation vgl. nur

Böckenförde (N 13), S. 80ff.; W. Henke, in: BK, Art. 21 Rn. 189.

Vgl. Thoma (N 59), S. 188.

Vgl. Stern (N 63), S. 894.

Vgl. Axel Hopfauf, Kein Präsentationsrecht bei Verfassungsrichterwahlen, in: ZRP 1994, S. 89 (90); zur Frage der Verzögerung noch u. Rn. $52 \mathrm{ff}$.

Dazu im einzelnen Häußler ( $N$ 65), S. 179ff.; für eine einfache Mehrheit nur im Vorbereitungsgremium etwa Billing (N 60), S. 311.

Karl-August Bettermann, "Die Aufgabe: Fachgericht für Verfassungsrecht", FAZ vom 20.Dez.1996, S. 13.

Vgl. dazu Claus Dieter Classen, Wahl contra Leistung? Zu Wahlbeamten und Richterwahlen, in: JZ 2002, S. 1009 (1013); Jochen Bleicken, Die athenische Demokratie, ${ }^{3} 1991$, S. 187, $218 f f ., 266$.

Vgl. etwa Preuß (N 61), S. 394; ausdrücklich anders etwa Billing (N 60), S. 308f.; dazu auch Häußler (N 65), S. 192. 


\section{DIE WAHL DER RICHTER AM DEUTSCHEN BUNDESVERFASSUNGSGERICHT ZWISCHEN RECHTLICHER THEORIE UND POLITISCHER PRAXIS}

Vgl. Preuß (N 61), S. 394.

Vgl. etwa Billing (N 60), S. 308, 313ff.; Stern (N 63), S. 895; Kröger (N 1), S. 99.

Vgl. zu den abschätzigen Begriffen etwa Geck (N 22), Rn. 14; Majer (N 19), § 6 Rn. 44. Vgl. etwa Gusy (N 28), § 60 Rn. 19; Geck (N 22), Rn. 17.

Vgl. schon im Titel Lamprecht (N 39), S. 314 ("Kungelei hinter den Kulissen").

Vgl. zur Kritik am Parteiproporz etwa Geck (N 22) Rn. 15; Stefan Ulrich Pieper, Verfassungsrichterwahlen, 1998, S. 36f.; Preuß (N 61), S. 392; Kröger (N 1), S. 92f.; verteidigend Gusy (N 28), § 60 Rn. $16 f$.

Zu diesem problematischen Begriff vgl. etwa Hans H. Klein, in: Maunz/Dürig, Komm. z. GG, Art. 21 Rn. 179ff.; Jörn Ipsen, in: Michael Sachs (Hg), GG: Kommentar, ${ }^{3} 2003$, Art. 21 Rn. 14; Rudolf Streinz, in: v. Mangoldt/ Klein/Starck, GG II, Art. 21 Abs. 1 Rn. 8, 24ff., 30; grundlegend, wenn auch in seiner Begriffsbestimmung heute nicht mehr prägend Gerhard Leibholz, Verfassungsrechtliche Stellung und innere Ordnung der Parteien, Verh. 38.DJT, 1951, C2.

Vgl. nur die Darstellung bei Streinz (N 83), Art. 21 Abs. 1 Rn. 24ff. m.w.N.

Vgl. Kischel (N 13), S. 109f. m.w.N.

BVerfGE 107, 339 (359).

Vgl. Ipsen (N 83), Art. 21 Rn. 14; Streinz (N 83), Art. 21 Rn. 30.

Vgl. Gerhard Leibholz, Volk und Partei im neuen deutschen Verfassungsrecht, in: DVBI. 1950, S. 194 (195).

Vgl. BVerfGE 107, 339 (361f.) (Sondervotum Hassemer, Broß, Osterloh); 20, 56 (100ff., 111); 1, 208 (225).

Vgl. etwa Klein (N 83), Art. 21 Rn. 208; Streinz (N 83), Art. 21 Abs. 1 Rn. 94f.; Henke (N 69), Art. $21 \mathrm{Rn}$. 181ff.; zur Geltung der Unabhängigkeit für die Amtsführung, nicht für die Amtsverleihung vgl. Häußler (N 65), S. 186.

Vgl. auch BVerfGE 20, 56 (101): "In der modernen Massendemokratie üben die politischen Parteien entscheidenden Einfluß auf die Besetzung der obersten Staatsämter aus. Sie beeinflussen die Bildung des Staatswillens, indem sie in das System der staatlichen Institutionen und Ämter hineinwirken, und zwar insbesondere durch Einflußnahme auf die Beschlüsse und Maßnahmen von Parlament und Regierung."

Diese Konsequenz war nicht zuletzt einigen kritischen Stimmen der 50'er Jahre bewußt, die aus Abneigung gegen den Einfluß der Parteipolitik einer Richterwahl durch die Legislative insgesamt ablehnend gegenüberstanden, vgl. dazu Kröger ( $N$ 1), S. 76f.; zur Unmöglichkeit einer Entpolitisierung der Wahl auch Gerd Roellecke, Zum Problem einer Reform der Verfassungsgerichtsbarkeit, in: JZ 2001, S. 115 (116); Gusy (N 28), § 60 Rn. 16; ähnlich Henke (N 69), Art. 21 Rn. 191.

Vgl. etwa Billing (N 60), S. 303, der fordert, daß bei der Wahl "das Parteipolitische vom Fachverstand überdeckt wird".

Vgl. Robert Alexy, Theorie der juristischen Argumentation, 1983, S. 17.

Zum Wertungsproblem vgl. etwa einerseits die an das Gesetz anknüpfende wertkritische Methode bei Helmut Coing, Grundzüge der Rechtsphilosophie, ${ }^{5} 1993$, S. 280f., 208f. sowie das an die Rechtsordnung angeknüpfte, wertorientierte Denken bei Karl Larenz, Methodenlehre der Rechtswissenschaft, ${ }^{6} 1991$, S. 214ff., 288ff.; andererseits die Herleitung von Werten vor allem auch aus der Natur des Menschen bei Heinrich Hubmann, Rationale Wertung im Recht, in: ders., Wertung und Abwägung im Recht, 1977, S. 1 (8, 14); grundlegend zur Logik der Werte Chaïm Perelman, Über die Gerechtigkeit, 1967, S. 135; empirische Untersuchung der Zusammenhänge von politischem Vorverständnis und Entscheidungen in den USA bei Cass R. Sunstein/David Schkade/Lisa Michelle Ellmann, Ideological Voting on Federal Courts of Appeal - A preliminary investigation, AEI-Brookings Joint Center for Regulatory Studies, Working Paper No. 03-9, 2003.

Vgl. Kischel (N 13), S. $12 f f$.

Vgl. Dieter Grimm, Politikdistanz als Voraussetzung von Politikkontrolle. Über die Unabhängigkeit des Verfassungsgerichts im Parteienstaat, in: EuGRZ 2000, S. 1 (2).

Vgl. Kischel (N 13), S. 9; zur Rationalität als Begründbarkeit vgl. Heino Garrn, Zur Rationalität rechtlicher Entscheidungen, 1986, S. $16 \mathrm{ff}$.

Vgl. zu den Grundrechten nur grundlegend Ernst-Wolfgang Böckenförde, Grundrechtstheorie und Grundrechtsinterpretation, in: ders., Staat, Verfassung, Demokratie, ${ }^{2} 1992$, S. 115 (115ff.); Ernst-Wolfgang Böckenförde, Die Methoden der Verfassungsinterpretation - Bestandsaufnahme und Kritik, in: ibd., S. 53 (53ff., insbes. 86ff.).

99. Vgl. Thoma (N 59), S. 171.

100. Vgl. zu diesem Aspekt der Wahl Hopfauf (N 72), S. 90. 
101. Anders offenbar Geck (N 22), Rn. 20 Fn. 28, der zwischen Ausgewogenheit und Parteienproporz unterscheidet; ähnlich wie hier Roellecke (N 92), S. 115f.

102. Vgl. nur Heinrich Wefing, Leuchtturm, in: FAZ v. 28. Dez. 2001, S. 53; Hans Vorländer, Der Interpret als Souverän, in: FAZ vom 17. April 2001, S. 14; die negative Prognose von Geck (N 3), S. 40f. hat sich insofern nicht bewahrheitet.

103. Vgl. auch schon o. Rn. 18; s. u. Rn. 72.

104. Vgl. etwa Ulrich Haltern, Demokratische Verantwortlichkeit und Verfassungsgerichtsbarkeit, in: Der Staat 36 (1996), S. 551 (554f.).

105. Immanuel Kant, Zum ewigen Frieden, 1795, in: Kant's Werke, Bd. 8, Neudruck 1923, Nachdruck 1969, S. 343 (381).

106. Detaillierte Darstellung bei Abraham (N 26).

107. Beispielsweise zur Abtreibung als etablierten Lackmustest republikanischer Präsidenten für die Nominierung vgl. Richard D. Manoloff, The Advice and Consent of The Congress: Toward a Supreme Court Appointment Process for our Time, in: 54 Ohio St. L.J. 1087, 1095-6 (1993).

108. Eine Möglichkeit vermehrter Ablehnungsanträge sieht deshalb Stern (N 63), S. 893; in Anbetracht der Befangenheitsregeln für wissenschaftliche Äußerungen dürfte davon aber kaum eine Gefahr ausgehen, vgl. dazu u. Rn. 58, 63, 66.

109. Zum original intent vgl. Laurence H. Tribe, American Constitutional Law, Bd. I, New York ${ }^{3} 2000$, S. 47ff.; vgl. auch Ulrich Haltern, Verfassungsgerichtsbarkeit, Demokratie und Mißtrauen, 1998, S. $245 \mathrm{ff}$.

110. Vgl. Zätzsch (N 26), S. $140 \mathrm{ff}$.

111. Abraham ( $\mathrm{N} 26)$, S. 298 spricht von "geschwollenem akademischen Diskurs" und von "gelehrten, vorlesungsähnlichen Antworten", Übersetzungen durch den Verf.

112. Kennedy führte aus: "Robert Bork's America is a land in which women would be forced into back alley abortions, blacks would sit at segregated lunch counters, rogue police could break down citizens' doors in midnight raids, school children could not be taught about evolution, writers and artists could be censored at the whim of government, and the doors of the federal courts would be shut on the fingers of millions of citizens for whom the judiciary is - and is often the only - protector of the individual rights that are the heart of our democracy.", zit. nach Stephen L. Carter, Bork Redux, or How the Tempting of America Led the People to Rise and Battle for Justice, in: 69 Tex. L. Rev. 759, 760-1 (1991).

113. So Carter (N 113), S. 762.

114. Zit. nach Abraham (N 26), S. 312; zum ganzen Verfahren vgl. ibd., S. 310ff.; Zätzsch (N 26), S. $142 f$.

115. Vgl. Manoloff (N 108), S. 1095.

116. Vgl. Lamprecht (N 56), S. 2533.

117. Vgl. Preuß (N 61), S. 395.

118. Vgl. Trautwein (N 60), S. 40; auf die Lage in der Schweiz weist insofern Majer (N 19), § 6 Rn. 48, 56 hin.

119. Vgl. Preuß (N 61), S. 394f.; Majer (N 19), § 6 Rn. 49.

120. Vgl. Preuß (N 61), S. 394f.; Majer (N 19), § 6 Rn. 49.

121. Vgl. William G. Ross, The Supreme Court Appointment Process: A Search for a Synthesis, in: 57 Alb. L Rev. 993, 994-5 (1994).

122. So auch Zätzsch (N 26), S. 151.

123. Die negativen persönlichen Auswirkungen auf unterlegene Kandidaten haben Verfassungsjuristen in den USA sogar schon zur literarischen Bearbeitung veranlaßt, vgl. Stephen L. Carter, The Emperor of Ocean Park, 2002.

124. In den USA unterliegt der angehörte Einzelkandidat statistisch in rund $20 \%$ der Fälle, vgl. Manoloff (N 108), S. 1088.

125. Zu einem zusätzlichen Argumentationsversuch über Art. 42 Abs. 2 GG, der wenig Widerhall gefunden hat, vgl. Johann-Friedrich von Eichborn, Die Bestimmungen über die Wahl der Bundesverfassungsrichter als Verfassungsproblem, 1969, S. 16, 21f., 38; dagegen überzeugend Kröger (N 1), S. 92.

126. Vgl. zum folgenden nur die Zusammenstellungen bei Koch (N 59), S. 42f.; Gusy (N 28), § 60 Rn. 12; v. Eichborn (N 126); S. $12 f f$.

127. So Thoma (N 59), S. 188.

128. Vgl. dazu auch schon die Bemerkungen des Bundesverfassungsgerichts zu dem Rechtsgutachten von Professor Richard Thoma, in: JöR 1957, S. 194 (202 Fn. 26).

129. Vgl. dazu etwa Majer (N 19), § 6 Rn. 35ff.; Klein (N 17), § 6 Rn. 3f.; Koch (N 59), S. 42, jeweils m.w.N.

130. Vgl. zu den Ausschüssen im allgemeinen etwa Jörn Ipsen, Staatsrecht I, ${ }^{15} 2003$, S. $80 f$. 


\section{DIE WAHL DER RICHTER AM DEUTSCHEN BUNDESVERFASSUNGSGERICHT ZWISCHEN RECHTLICHER THEORIE UND POLITISCHER PRAXIS}

131. Vgl. dazu nur Hartmut Bauer, in: Dreier, GG II, Art. 50 Rn. 18 m.w.N.

132. Vgl. dazu Ernst-Wolfgang Böckenförde, Demokratie als Verfassungsprinzip, in: ders., Staat, Verfassung, Demokratie, ${ }^{2} 1992$, S. 289 (301).

133. Vgl. BVerfGE 80, 188 (221f.).

134. Vgl. BVerfGE 88, 188 (225, vgl. auch 230).

135. BVerfGE 88, $188(220)$.

136. Vgl. BVerfGE 44, 308 (315).

137. Vgl. BVerfGE 44, 308 (317).

138. Vgl. BVerfGE 44, 308 (317)

139. So BVerGE 80, 188 (243) (Sondervotum Kruis).

140. Vgl. für diese Tendenz etwa Preuß (N 61), S. 389ff., der etwa von einer "quasiparlamentarischen Leitungsfunktion des Bundesverfassungs'gerichts'" spricht, ibd., S. 391 (Hervorhebung und Anführungszeichen im Orig.).

141. Dazu und zum Problem allgemein Böckenförde (N 46), S. 11f.; vgl auch Ernst-Wolfgang Böckenförde: Die Eigenart des Staatsrechts und der Staatsrechtswissenschaft, in: ders., Staat, Verfassung, Demokratie, ${ }^{2} 1992$, S. 11 (15f.).

142. Vgl. Kischel (N 13), S. 7.

143. Vgl. Haltern (N 105), S. 551ff.; Haltern (N 110), S. $73 f f$.

144. Zum Begriff des formalism im US-Recht vgl. kurz Uwe Kischel, Delegation of legislative power, in: ALR 1994, S. 213 (249) m.w.N.

145. Haltern (N 110), S. 73ff.; vgl. aus politikwissenschaftlicher Sicht auch Hans Vorländer, Der Interpret als Souverän, in: FAZ vom 17. April 2001, S. 14 ("Verfassungsgerichtsbarkeit (...) lebt gerade von den fiktionalen Formen, daß es sich (...) um die bloße Anwendung der Verfassung (...) handelt").

146. Vgl. Grimm (N 97), S. 2 ("Deswegen verläuft die Diskussion auch in den Bahnen der juristischen Argumentationsstandards [...]. [...] würde doch derjenige, der diese [bestehenden Interpretations-]Spielräume mit politischen Argumenten füllen wollte, kein Gehör finden. Die Bindung an einen normativen Text, an die juristische Interpretationsmethode und an die vorausgegangenen Entscheidungen des Gerichts dürfen nicht unterschätzt werden. Sie führen zu einer Brechung der außer- und vorjuristischen Überzeugungen und Verständnisse, von denen selbstverständlich kein Richter frei ist."); Paul Kirchhof, Die Verfassung wahrt Rechtserinnerung und Nachdenklichkeit, in: FAZ v. 12.2.2000, S. 8 ("ist das Gericht der dank Wortgebundenheit nachdenkliche Vorsprecher des Verfassungsrechts"); Böckenförde ( $N$ 46), S. 11 f. ("Bindung an einen rational nachprüfbaren, methodisch abgesicherten Argumentationszusammenhang", "Diese Streitigkeiten hören deshalb allerdings nicht auf, rechtliche Streitigkeiten zu sein, die allein nach einem Rechtsmaßstab zu entscheiden sind").

147. Geck (N 22), Rn. 18 sieht einen Zusammenhang zwischen Unkenntnis der Öffentlichkeit von der Wahlpraxis und hohem Ansehen des Gerichts.

148. Dazu Thomas Oppermann, Das Bundesverfassungsgericht und die Staatsrechtslehre, in: FS 50 Jahre BVerfG, Bd. 1, 2001, S. 421 (421ff.).

Recebido para publicação: 05/12/2011

Aceito para publicação: 23/12/2011 